\title{
SAR IMAGE COMPRESSION USING ADAPTIVE DIFFERENTIAL EVOLUTION AND PATTERN SEARCH BASED K-MEANS VECTOR QUANTIZATION
}

\author{
KARRI CHIRANJEEVI $^{\bowtie, 1}$, UMARANJAN JENA $^{2}$ \\ ${ }^{1}$ Department of Electronics and Communication Engineering,' GMR Institute of Technology, Rajam, \\ Srikakulam, Andhrapradesh, India; ${ }^{2}$ Department of Electronics and Telecommunication Engineering, Veer \\ Surendra Sai University of Technology (VSSUT), Burla-768018, Odisha, India. \\ e-mail: chiranjeevi.k@gmrit.org, urjena@rdiffmail.com² \\ (Received August 26, 2016; revised August 18, 2017; accepted August 21, 2017)
}

\begin{abstract}
A novel Vector Quantization (VQ) technique for encoding the Bi-orthogonal wavelet decomposed image using hybrid Adaptive Differential Evolution (ADE) and a Pattern Search optimization algorithm (hADEPS) is proposed. ADE is a modified version of Differential Evolution (DE) in which mutation operation is made adaptive based on the ascending/descending objective function or fitness value and tested on twelve numerical benchmark functions and the results are compared and proved better than Genetic Algorithm (GA), ordinary DE and FA. ADE is a global optimizer which explore the global search space and PS is local optimizer which exploit a local search space, so ADE is hybridized with PS. In the proposed VQ, in a codebook of codewords, $62.5 \%$ of codewords are assigned and optimized for the approximation coefficients and the remaining $37.5 \%$ are equally assigned to horizontal, vertical and diagonal coefficients. The superiority of proposed hybrid Adaptive Differential Evolution and Pattern Search (hADE-PS) optimized vector quantization over DE is demonstrated. The proposed technique is compared with DE based VQ and ADE based quantization and with standard LBG algorithm. Results show higher Peak Signal-to-Noise Ratio (PSNR) and Structural Similiraty Index Measure (SSIM) indicating better reconstruction.
\end{abstract}

Keywords: differential evolution (ADE); image compression; Linde-Buzo-Gray (LBG); Pattern Search (PS); vector quantization.

\section{INTRODUCTION}

Synthetic Aperture Radar (SAR) images are high resolution images that carries the amplitude and phase information of object/target captured and processes with transferable radar. These are beneficial for military applications, plant investigations, remote sensing and Earth scientists. Transmitting or storing of SAR images requires large time and high data storage devices, consequently necessitates the use of efficient and effective image compression techniques. SAR image has less spatial correlation, identifying homogeneous regions and high dynamic range (Zeng and Cumming, 2001) is difficult as compared to ordinary optical images. So the compression techniques to solve an ordinary optical image are not suitable for compression of SAR images. SAR Image compression plays crucial role in the field of multimedia applications, streaming data on the Internet, data storage, wire and wireless communication, chip designing and computer to computer communication.
Image Compression is a technique of representating the image that results in reduction of bits per pixel and processing time. Image compression is performed in three processing stages: pixel transforms, Quantization and entropy coding. Pixel transformation is a powerful process for image compression where spatial domain image is transformed into a frequency domain image with some transformation techniques like DWT (JPEG-2000 and JPEG-XR), DCT (JPEG,). In this work the focus is on Quantization. It has two variants, one is a scalar, applicable for one dimensional (speech, voice) and another is vector quantization/block quantization/pattern matching applicable for two dimensions (image). According Shannon's rate-distortion information theory, compression with vector quantization performance is better to scalar quantization (Liu and Ling, 2015).Vector quantization is a process of representing high two dimensional vectors/image into a low dimensional vector/image (called codebook) based on the minimum Euclidean distance/ average distortion. The elements in the codebook are called 
codewords. The minimization of the average distortion is a nonlinear problem, which is generally achieved by a gradient-descent-based iterative procedure called the generalized Lloyd algorithm (GLA)/LBG Algorithm (Linde et al., 1980). The goal of VQ is to find a codebook which minimizes the Euclidean distance between training vector and codewords. LBG algorithm is simple, adaptable and flexible, but it suffers with local optimal problem. The LBG algorithm does not guarantee the global best solution since its solution depends on the initial solution of the algorithm. So Patane et al. (2002) proposed an enhanced LBG algorithm that avoids the local optimal problem. In genetic algorithm, crossover and mutation plays vital role. Krishna et al. (1997) obtained optimal fast codebook with genetic algorithm by applying genetic algorithm on k-means clustering with the help of Gradient descent in which k-means algorithm is used as crossover operation and mutation operation is distance based. Chen (2012) hybridized the Genetic algorithm and LBG algorithm for optimizing the center of clusters. Zheng et al. (1997) applied genetic algorithm to speed up the LBG algorithm by using an appropriate fitness function. The results obtained are compared with those of non-genetic algorithm and proved effective but time consuming.

George and Dimitrios (2012) incorporated the cmeans and the fuzzy c-means in a uniform fashion for balancing the speed and efficiency of vector quantization. Zhao and Liu (2013) presented a clonal cluster method based on the manifold distance, which produces a final codebook with the help of optimization technique. Horng and Jiang (2011) proposed an Artificial Bee Colony (ABC) based VQ that shows improvement in PSNR with good reconstructed image quality as compared to LBG, PSO, QPSO and HBMO. Rajpoot et al. (2004) designed a codebook by vector quantizing the DWT transformed wavelet coefficients with the help of Ant Colony Optimization (ACO) algorithm. They designed a codebook by identification of the edges of the graph and arranging the wavelet coefficients in a bidirectional graph. It was found that quantization of zero-tree vectors using ACO outperforms LBG algorithm, but ACO convergence time is high. Tsaia et al. (2013) observed that during the convergence process of ACO for Codebook Generation Problem (CGP), patterns or sub-solutions reach their final states at different times. Also, most of the patterns are assigned to the same codewords after a certain number of iterations. Particle Swarm Optimization (PSO) vector quantization, based on updating the global best (gbest) and particle best (pbest) solution (Chen et al., 2005) outperforms LBG algo- rithm. The gbest holds highest fitness value among all populations and pbest holds the best fitness value of corresponding particle. The Feng et al. (2007) suggested Evolutionary fuzzy particle swarm optimization algorithm which is a combination of PSO and adaptive Fuzzy Inference Method (FIM) to obtain better global performances than LBG learning algorithms.

Quantum Particle Swarm Algorithm (QPSO) was proposed by Wang et al. (2007) to solve the 0-1 knapsack problem. The QPSO performance is better than PSO; it computes the local points from the pbest and gbest for each particle and updates the position of the particle by choosing appropriate parameters $u$, a random number that lies between 0 and 1 and $z$ which is non-negative constant and is less than 2.8. Poggi et al. (2001) proposed Tree-structured product-codebook vector quantization, which reduces encoding complexity even for large vectors by combining the tree-structured component codebooks and a low-complexity greedy procedure. Hu et al. (2008) proposed a fast codebook search algorithm based on triangular inequality estimation. Sanyal et al. (2013) applied a new approach for the selection of chemotaxis steps of basic Bacterial Foraging Optimization Algorithm (BFOA) which leads to the development of a near optimal codebook for image compression with good reconstructed image quality and high peak signal to noise ratio. Fuzzy membership function is optimized by the modified Bacterial Foraging Optimization and compared the results with other optimization techniques. Horng et al. (2011) applied honey bee mating optimization algorithm for Vector quantization. HBMO has high quality reconstructed image and better codebook with small distortion compared to PSO-LBG, QPSO-LBG and LBG algorithm.

Horng (2012) applied a Firefly Algorithm (FA) to design a codebook for vector quantization. The firefly algorithm has become an increasingly important tool of Swarm Intelligence that has been applied in almost all areas of optimization, as well as engineering practice. Firefly algorithm is encouraged by social activities of fireflies and the occurrence of bioluminescent communication. Fireflies with lighter intensity values move towards the brighter intensity fireflies and if there is no brighter firefly then it moves randomly. Objectbased VQ was proposed by Abouali (2015) based on an iterative process of LBG algorithm, max-min algorithm and multi-object applications. The proposed method takes the advantage of well suitable high dimensional problem technique which is an adaptive differential evolution (ADE) and near around/local search technique which is pattern search (PS) for 
effective codebook design by taking the initial solution of K-Means clustering algorithm/LBG Algorithm as one of the population/solution. In this work the modified version of differential evolution and pattern search are cascaded to form a Hybrid ADEPS (hADE-PS) algorithm. The SAR image to be vector quantized is transformed to wavelet domain with the help Biorthogonal Discrete Wavelet Transform (DWT) because most of the energy is concentrated on the low frequency band. The transformed SAR image is now vector quantized by using K-Means clustering algorithm on which hADE-PS works for efficient codebook design. Adaptive differential evolution is developed and tested on benchmark functions and results obtained are verified and found to be better as compared to ordinary differential evolution in which mutation operation is random. Whereas ADE follows a specific order/strategy while selecting population for mutation operation. To improve compression ratio with considerable PSNR and reconstructed image quality, a perfect codebook design is crucial for the researcher. So in this paper, a hybrid adaptive differential evolution and pattern search is proposed for efficient codebook design by optimizing the solution of K-Means clustering algorithm. The optimized codebook and corresponding index table values are further coded with a run length coding followed by a Huffman coding at the transmitter section and reverse operation at the receiver section. The proposed method is compared with the Differential Evolution (DE) based VQ and Adaptive Differential Evolution (ADE) based VQ and experimentally proved that it has superior Peak Signal Noise Ratio (PSNR), Mean Square Error (MSE), fitness function, bits per pixel, compression ratio and Structural Similiraty Index Measure (SSIM). This paper is organized in five sections including the introduction. In section 2, proposed framework for SAR Image compression and recent methods of VQ is discussed along with their algorithms. The proposed method of hybrid adaptive differential evolution and pattern search VQ algorithm is presented with the procedure in section 3. The results and discussions are given in section 4. Finally the conclusion is given in section 5 .

\section{MATERIALS AND METHODS}

\section{CONTRIBUTION}

In the proposed method of SAR image compression, the differential evolution is modified as adaptive differential evolution for efficient codebook design and for further improvement a pattern search algorithm is applied on the solution of adaptive differential evolution. The encoding and decoding procedure of proposed SAR image compression is shown in Fig. 1. At the transmitter/encoding section, the SAR image to be vector quantized for compression is transformed to wavelet domain by using Biorthogonal Discrete Wavelet Transform and further partitioned into non overlapping blocks called input vector. The input vectors are clustered based on the minimum Euclidean distance between the input vector and codewords of codebook by means of iterative method called Kmeans clustering algorithm/LBG algorithm. The obtained codebook is optimized by the proposed hybrid adaptive differential evolution and pattern search algorithm. The optimized codebook of index table contains repeated information, so it is coded by variable Run-length coding followed by Huffman coding. At the receiver/decoder section, the index values are retrieved by Huffman decoding and Runlength decoding and from these index values the corresponding codewords are obtained from the receiver end codebook and rearranged to get a reconstructed/decompressed image.
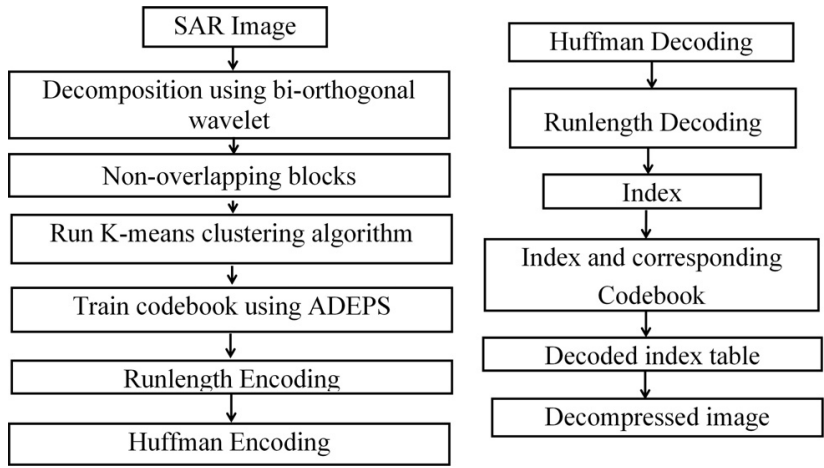

Fig. 1. Encoding and decoding process of proposed vector quantization.

\section{VECTOR QUANTIZATION}

The vector quantization is a one of the block coding technique for image compression. Codebook design is an important task in the design of VQ that minimize the distortion between reconstructed image and original image with less computational time. Fig. 2 shows the encoding and decoding process of vector quantization. The image (size $N \times N$ ) to be vector quantized is subdivided into $N_{b}(N / n \times N / n)$ blocks with size $n \times n$ pixels. These divided image blocks or training vectors of size $n \times n$ pixels are represented with $X_{i}\left(i=1,2,3, \ldots, N_{b}\right)$. The Codebook has a set of codewords, $C_{i}$ (where $i=1 \ldots N_{c}$ ) is the $i^{\text {th }}$ codeword. The total number of codewords in Codebook is $N_{c}$. Each subdivided image vector is approximated by the index of codewords based on the minimum Euclidean distance between corresponding vector and code 
words. The encoded results are called an index table. During the decoding procedure, the receiver uses the same codebook to translate the index back to its corresponding codeword for reconstructing the image. The distortion/fitness function $(D)$ between training vectors and the codebook is given as

$D=\frac{1}{N_{c}} \sum_{j=1}^{N_{c}} \sum_{i=1}^{N_{b}} u_{i j} \cdot\left\|X_{i}-C_{j}\right\|^{2}$.

Subject to the following constraints:

$D=\sum_{j=1}^{N_{c}} u_{i j}=1 \quad \mathrm{i} €\left\{1,2, \ldots, \mathrm{N}_{\mathrm{b}}\right\}$,

$u_{i j}$ is one if $X_{i}$ is in the $j^{\text {th }}$ cluster, otherwise zero.

Two necessary conditions exist for an optimal vector quantizer.

(1) The partition $R_{j}, j=1, \ldots, N_{c}$ must satisfy $R_{j} \supset\left\{x \varepsilon X: d\left(\mathrm{c}, \mathrm{C}_{j}\right)<d\left(\mathrm{x}, \mathrm{C}_{k}\right), \forall \mathrm{k} \neq \mathrm{j}\right\}$.

(2) The codeword $C_{j}$ must be given by the centroid of $R_{j}$ :

$$
C_{j}=\frac{1}{N_{j}} \sum_{i=1}^{N_{j}} x_{i} \quad x_{i} \in R_{j},
$$

where $N_{j}$ is the total number of vectors belonging to $R_{j}$

\section{DECOMPOSITION USING BIORTHOGONAL WAVELET}

In 1970's images are decomposed with Discrete Cosine Transform (DCT) in which most of the energy is concentrated in DC coefficients, that helps for high compression with considerable artifact effect. The image compression has leaped to a new level (Daubechies, 1988) with the introduction of Discrete Wavelet Transform (DWT). Unlike DCT, the DWT provides both spatial and frequency information about the image. The DWT decomposes the image into four coefficients; approximation (low-low frequency), horizontal (low-high frequency), vertical (high-low frequency) and diagonal (high-high frequency) coefficients. These coefficients are obtained with the parallel combination of low pass filter and high pass filter and down samplers as shown in Fig. 3. Fig. 4 shows the three dimensional view of approximation, horizontal, vertical and diagonal coefficients of a Flight Line SAR image. It is observed that approximation coefficients carry much information about the input image as compared to other coefficients whereas all horizontal, vertical and diagonal coefficients spread their values near around to particular values, that helps for good clustering results in better image compression as shown in Fig. 4d. For the sake of fidelity of reconstructed image quality one level decomposition is applied, the same can be applied to more than one decomposition levels for a high degree of compression at the cost of time. Like in JPEG2000 , the wavelet used in our work for decomposition is biorthogonal wavelet because of its simple design and option to build symmetric wavelet functions. In the proposed method, optimization technique spent much time in the codebook design of approximation coefficients and less time for remaining, because the reconstructed image quality depends predominantly on approximation coefficients.

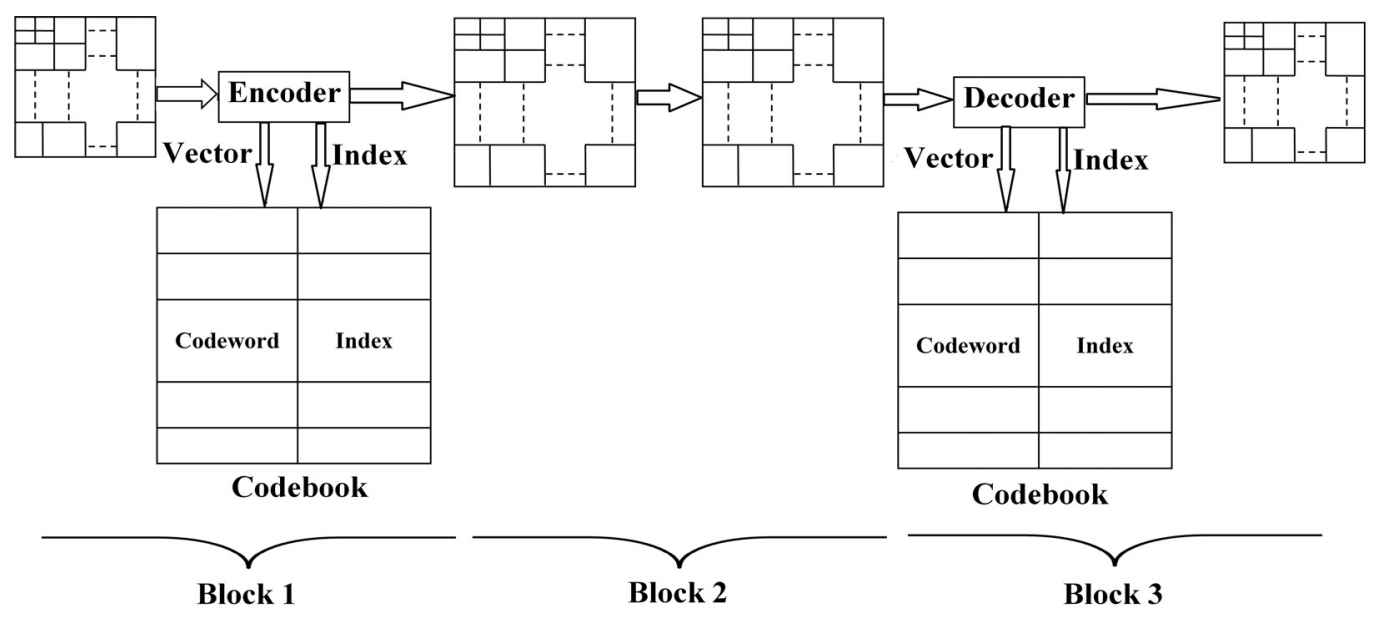

Fig. 2. Encoding and decoding process of vector quantization. 


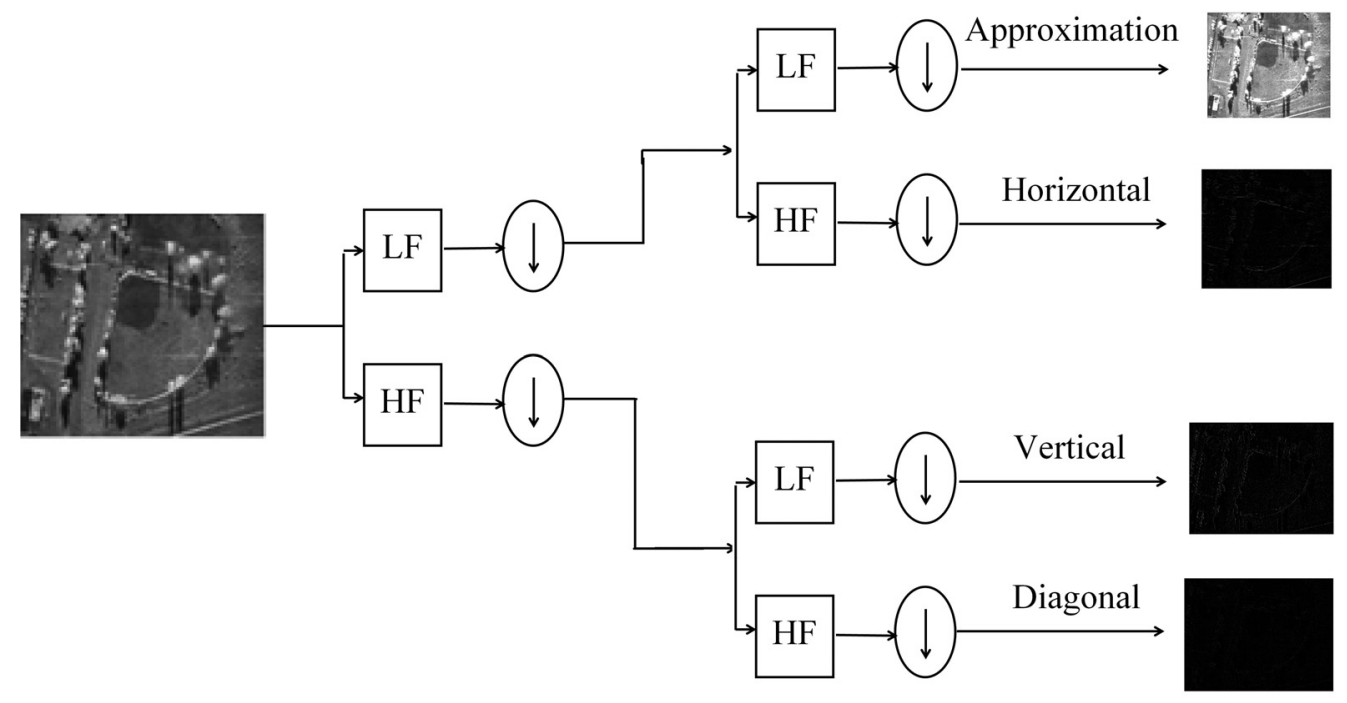

Fig. 3. Wavelet decomposition.
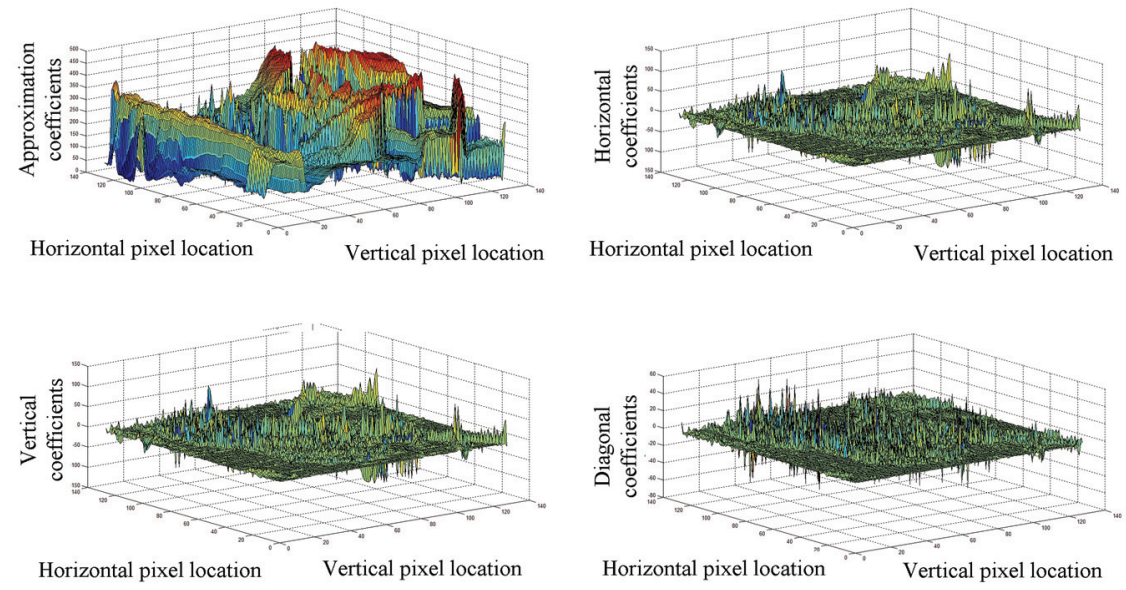

Fig. 4. Three dimensional view of Approximation, Horizontal, Vertical and Diagonal coefficients of a Flight Line SAR image.

In a codebook of eight codewords, using the proposed VQ, five codewords are assigned and optimized for the approximation coefficients, one for horizontal, one for vertical and one for diagonal coefficients. Similarly, in a codebook size of 16, 32, 64, 128, 256 and 512, approximation coefficients takes codewords of $10,20,40,80,160$ and 320 respectively and remaining codewords $6,12,24,48,96,192$ are assigned for the other three coefficients with each containing a codeword of $2,4,8,16,32,64$ respectively. While optimizing the codebook population/ solution, maximum and minimum values are selected based on the max and min value of corresponding coefficients. After successful codebook optimization with the proposed hADE-PS, index table and codebook are coded by Run Length Coding (RLE) and Huffman coding. Runlength coding is a lossless coding, aiming to reduce the amount of data needed for storage and transmission. It represents consecutive runs of the same value in the data as the value, followed by the count or vice versa. If all the values in the original data are same, RLE can reduce data to just two values otherwise double of the original data. Therefore, RLE should only be used in cases where runs of the same value are expected. Huffman coding is a lossless variable length coding, best fit for compressing a data/ image obtained from run length coding. It uses fewer bits to represent frequent symbols and more bits to represent infrequent symbols. The performance of Huffman coding purely depends on the effective development of Huffman tree with minimum weighted path length. The time complexity of Huffman coding is $\mathrm{O}\left(\mathrm{Nlog}_{2} \mathrm{~N}\right)$ where each iteration $\mathrm{O}\left(\log _{2} N\right)$ time to determine the cheapest weight and there would be $O$ $(N)$ iterations. 


\section{GENERALIZED LBG VECTOR QUANTIZATION ALGORITHM}

The most commonly used methods in VQ are the Generalized Lloyd Algorithm (GLA) which is also called Linde-Buzo-Gary (LBG) algorithm. The algorithm is as follows:

Step 1: Begin with initial codebook $C_{l}$ of size $N$. Let the iteration counter be $m=1$ and the initial distortion $D_{l}=\infty$.

Step 2: Using codebook $C_{m}=\left\{Y_{i}\right\}$, partition the training set into cluster sets $R_{i}$ using the nearest neighbor condition.

Step 3: Once the mapping of all the input vectors to the initial code vectors is made, compute the centroids of the partition region found in step 2. This gives an improved codebook $C_{m+1}$.

Step 4: Calculate the average distortion $D_{m+l}$. If $D_{m}-D_{m+1}<T$ then stops, otherwise $m=m+1$ and repeat step 2-4.

\section{HYBRID ADAPTIVE DIFFERENTIAL EVOLUTION AND PATTERN SEARCH (HADE-PS) VQ ALGORITHM}

To examine the extremely multimodal space, a two phase hybrid method recognized as hybrid Differential Evolution and Pattern Search (hADE-PS) is employed. In this algorithm, DE is used for global exploration and the pattern search is employed for local search. The first phase is explorative, employing a classical ADE to identify hopeful areas of the search space. The best solution initiated by ADE is then polished using the PS method during a consequent exploitative phase. In order to find the advantage of projected hADE-PS approach, the results are compared with the DE algorithm. In the subsequent section, proposed $\mathrm{ADE}$ is employed for for efficient codebook design.

\section{DIFFERENTIAL EVOLUTION VECTOR QUANTIZATION}

The LBG algorithm distortion becomes smaller after recursive execution. Actually, the LBG algorithm can guarantee that the distortion will not increase from one iteration to the next iteration. However, the resulting codebook may not be the optimum one and the initial condition will significantly influence the results (Chiranjeevi and Umaranjan, 2015). Therefore, in the LBG algorithm more attention should be given to the choice of the initial codebook. The problems with the LBG algorithm can be overcome by using differential evolution. Differential evolution is metaheuristics algorithm introduced for large dimensional problems in the year 1995 by (Stron and price, 1995). Differential evolution is applicable in the field of engineering and science problems because of its high speed of convergence, less expensive, easy to implement, negligible parameter tuning and real coding. The working process of DE is similar to the GA in which three steps are crossover, mutation and selection. The DE algorithm initializes the populations/solutions $X$ $\left(X_{1}, X_{2}, X_{3}\right.$,----- $\left.X_{N}\right)$ of size $N$ and $D$ dimensions in between upper and lower limits of the problem $(0,255)$, in this paper $D$ is equal to 16 (block size $=$ 16). The population values are initialized randomly within the limited upper $\left(X_{\text {upper }}\right)$ and lower $\left(X_{\text {lower }}\right)$ bounds as given in Eq. 5, here as the image is grayscale image upper bound is 255 (white) and lower is 0 (black).

$$
\begin{array}{r}
X_{K}=X_{\text {lower }}+\operatorname{rand}(1,1) \times\left(X_{\text {upper }}-X_{\text {lower }}\right) \\
K=1,2,3,---N,
\end{array}
$$

where rand $(1,1)$ is a random number of size one lying between 0 and 1

Calculate the fitness/objective function of all populations $\left(\mathrm{f}\left(\mathrm{X}_{1}\right), \mathrm{f}\left(\mathrm{X}_{2}\right), \mathrm{f}\left(\mathrm{X}_{3}\right)\right.$,------- $\left.\mathrm{f}\left(\mathrm{X}_{\mathrm{N}}\right)\right)$ and considered these as old generation. In each iteration the new generation of same population is generated with three steps: mutation, crossover and selection. In mutation operation, for all populations in each iteration a trial vector/donor vector $V_{K}$ is created by adding the weighted difference of randomly selected two populations multiplied by the scalar multiple control parameter $(F)$ to a random third population as given in Eq. 6, these three populations are selected randomly from the initialized populations.

$$
V_{k}^{i}=X_{r 1}{ }^{i}+F \times\left(X_{r 2}{ }^{i}-X_{r 1}{ }^{i}\right),
$$

where $r_{1}, r_{2}$ and $r_{3}$ are randomly selected numbers lying between 1 and $N, \mathrm{i}$ is the $\mathrm{i}^{\text {th }}$ iteration and $\mathrm{F}$ is scaling factor lying between 0 and 2 . The next step of $\mathrm{DE}$ is crossover; to strengthen the potential diversity of solution and to generate a new solution called target vector. DE offers two kinds of crossovers named Exponential and Binomial, among two we did with Binominal in which for each trial vector an offspring vector $U_{K}$ is created as in Eq. 7, if the generated random number is less than the control parameter crossover rate $(C R)$. The crossover rate lies between 0 and 1 . DE offers several variants or mutation strategies like DE/rand/1, DE/best/2 etc... (Price et al., 2005). The last stage of $\mathrm{DE}$ is the selection process for maintaining the constant population size in successive generations and it selects either target 
vector or trial vector in the next generation based on their fitness value which follows the Darwinian principle given in Eq. 8.

$$
\begin{aligned}
U_{k} & = \begin{cases}V_{K}, & \text { if rand }(0,1)<C R, \\
X_{K}, & \text { Else }\end{cases} \\
X_{K}(t+1) & = \begin{cases}V_{K}(t), & \text { if } f\left(U_{K}(t)\right) \leq f\left(X_{K}(t)\right) \\
X_{K}(t), & \text { if } f\left(U_{K}(t)\right)>f\left(X_{K}(t)\right)\end{cases}
\end{aligned}
$$

DE vector quantization algorithm:

Step 1: Initialize population size $(N)$, scaling factor $(F)$, crossover constant $(C R)$, maximum number of iterations, tolerance, lower limit and upper limit.

Step 2: Evaluate the fitness of the population using Eq. 1 and generate a trial vector $V_{K}$ with the help of Eq. 6.

Step 3: Crossover operation on population with crossover constant and generation of target vector $U_{K}$ with the help of Eq. 7.

Step 4: Replace the old generation with newly generated population which are generated based on the fitness value of target vectors $U_{K}$ or $X_{K}$.

Step 5: repeat step 2 to 4 until stopping criteria.

\section{PATTERN SEARCH VECTOR QUANTIZATION}

Hooke and Jeeves (1960) developed a Pattern search algorithm in the year 1960 for getting a solution of real time numerical and engineering problems which are unsolved by classical methods. Pattern search doesn't require the gradient of the problem to be optimized, so it is a derivative free or direct search optimization technique. Pattern search optimizes the objective function with two types of moves. First step is an exploratory move where, step of the movement of the solution is small in the direction of low/high objective function values and second is pattern move where, step of the movement of the solution is large in the direction of low/high objective function values.

Let starting point $X^{(0)}$, acceleration factor $a$, perturbation vector $P_{0}$ and perturbation tolerance vector $T$. Initialize the current perturbation vector: $P$ $\leftarrow P_{0}$. Find new solution $X^{(l)}$ by exploratory search around $X^{(0)}$. If $X^{(1)}$ fitness value is not better than $X^{(0)}$ then, Reset all of the perturbations to $1 / 2$, i.e., $P \leftarrow P / 2$. If any member of $P$ is now smaller than its corresponding perturbation tolerance in $T$, then exit with $x^{(0)}$ as the solution. If $X^{(l)}$ fitness value is not better than
$X^{(0)}$ then, reset the perturbation vector to its original value, i.e., $P \leftarrow P_{0}$ and follow the Pattern Move. Pattern move generate $X^{(2)}$ from $X^{(0)}$ through $X^{(1)}$ which is given by $X^{(2)}=X^{(0)}+a \times\left(X^{(1)}-X^{(0)}\right)$. Now apply exploratory search on $X^{(2)}$ and find the fitness $f\left(X^{(2)}\right)$, if it is not better than $f\left(X^{(1)}\right)$ then replace solution $X^{(0)}$ with solution $X^{(1)}$. If $f\left(X^{(2)}\right)$ is better than or equal to $f\left(X^{(l)}\right)$ then, replace solution $X^{(0)}$ with solution $X^{(1)}$ and $X^{(1)}$ with solution $X^{(2)}$. In this paper the initial solution $X^{(0)}$ is the outcome of the Adaptive differential evolution. At the initial iteration, perturbation vectors are initialized with the positions [0.5 $0.5],\left[\begin{array}{ll}0.5 & -0.5\end{array}\right],\left[\begin{array}{ll}-0.5 & 0.5\end{array}\right]$ and $\left[\begin{array}{ll}-0.5 & -0.5\end{array}\right]$ with acceleration factor is equal to one. To generate a next solution $X^{(l)}$ those obtained from the perturbation vectors are added to $X^{(0)}$ i.e $X^{(0)}+[0.50 .5], X^{(0)}+[0.5$

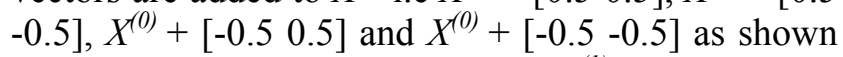
in Fig. 5. Calculate the fitness of $X^{(l)}$, if better than $X^{(0)}$ then perturbation vectors are consi-dered as successful perturbation vectors and algorithm replace the $X^{(0)}$ with $X^{(1)}$. Whenever successful per-turbation vectors occurred the algorithm will shift its state to pattern move where the acceleration factor is multiplied by a factor 2 so acceleration factor is also called as expansion factor. In the next iteration the new solution is $X^{(l)}+2 \times\left[\begin{array}{ll}0.5 & 0.5\end{array}\right], X^{(l)}+2 \times\left[\begin{array}{ll}0.5 & -0.5\end{array}\right], X^{(l)}+$ $2 \times\left[\begin{array}{ll}-0.5 & 0.5\end{array}\right]$ and $X^{(1)}+2 \times[-0.5-0.5]$ this process is repeated until stopping criteria/maximum iteration. In process, if any perturbation vectors resultant fitness value not better than the initial/current fitness than perturbation vectors are called unsuccessful and the same solution carried to the next iteration. In this situation the algorithm acceleration factor is divided by a factor 2 so called contraction factor.

Pattern search vector quantization algorithm:

Step 1: Initialize number of iterations, dimensions of the problem, mesh contraction factor/mesh expansion factor $(P)$, solutions $(K)$.

Step 2: check the convergence for all possible solutions $\left(\mathrm{X}_{\mathrm{k}}\right)$ where $k=1,2,3, \ldots \ldots K$.

Step 3: Calculate the objective function $f\left(X_{k}\right)$, and with the help of exploratory move calculates the step of search $S_{K}$.

Step 4: If new objective function $f\left(X_{k}+S_{k}\right)$ is less than $f\left(X_{k}\right)$, then new solution $X_{k+1}=X_{k}+S_{k}$ otherwise $X_{k+1}=X_{k}$.

Step 5: update mesh contraction factor/mesh expansion factor $(P)$. 


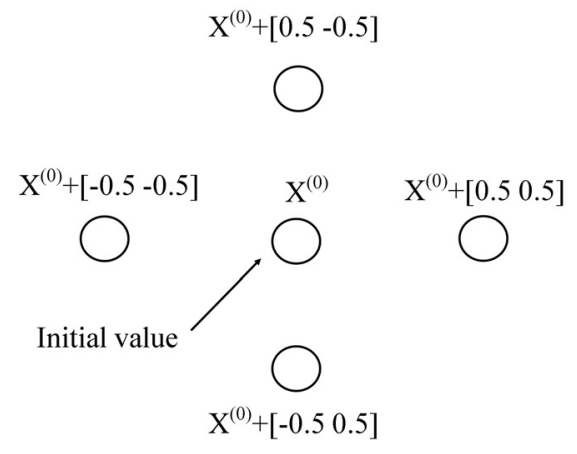

Exploratory move state/First iteration

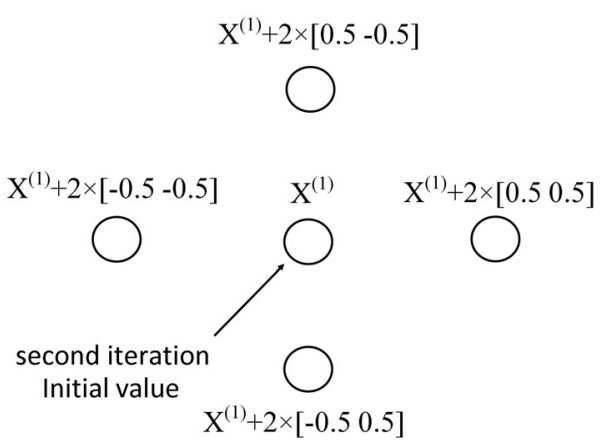

pattern move state

Fig. 5. Exploratory move state and pattern move state of pattern search.

\section{ADAPTIVE DIFFERENTIAL EVOLUTION}

The success of DE in solving a specific problem crucially depends on appropriately choosing donor vector generation strategies. In ordinary differential evolution, for a donor vector generation the selection of three populations is random as in Eq. 5, that results in a local optimal solution because the difference of two randomly selected populations nearly equals to largest solution and in addition to it the added population to the above difference results in a value that lies out of search space. As there is no particular pattern being followed in the selection of populations, it results in local optimal solution. So to overcome this we propose an adaptive differential evolution, to improve the performance, effectiveness and robustness of differential evolution for efficient vector quantization of image which leads to better image compression with good reconstructed image quality. The proposed novel differential evolution follows an intelligent selection of the population for donor vector $V_{K}$ that reduce the distortion $(D)$ between image to be compressed and codebook which is to be optimized. The ADE divides the population into two equal groups based on the descending fitness values. The first group is exploited for local search of the codebook, as the populations are arranged in descending order, the difference magnitude of two successive populations is less, which is added to the predecessor population. Hence the predecessor population explores for a solution around it as shown in Fig. 6. The same procedure is repeated for the first $N / 2$ populations among the available $\mathrm{N}$ descended populations.

For the global search of the codebook both the groups are harnessed. The donor vector generation is obtained from the difference of least fitness population
( $N^{\text {th }}$ element - second group) and the highest fitness population $\left(1^{\text {st }}\right.$ element - first group $)$ is added to $(N-$ 1) ${ }^{\text {th }}$ population. Similarly

The difference of the next least fitness population $\left((N-1)^{\text {th }}\right.$ element - second group) and the precedent highest fitness population ( $2^{\text {nd }}$ element - first group) is added to $(N-2)^{\text {th }}$ population and the same procedure is repeated for the remaining populations as depicted in the Fig. 6.

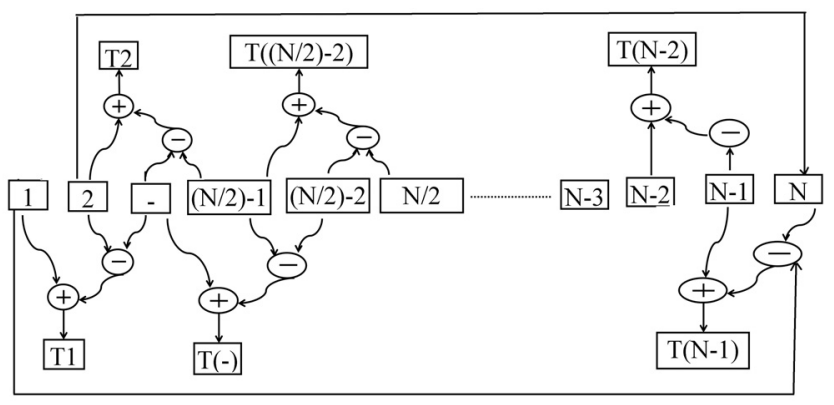

Fig. 6. Diagrammatic representation of proposed $A D E$.

The donor vector for $N^{\text {th }}$ population is generated on the same lines as that of ordinary differential evolution. This complete process is repeated for various iterations until distortion between codebook and image to be compressed is a global minimum. To validate the performance of $\mathrm{ADE}$, it is compared with $\mathrm{DE}$ based on nine benchmark functions. The simulation results show that ADE promises competitive performance not only in the PSNR, but also in the quality of reconstructed images. The algorithm of ADE is as similar as DE except the selection of three populations in a donor vector generation. The idea of using an adaptive selection of populations is to make ADE computationally more efficient in obtaining local and global solutions for the codebook. 
Table 1. Simulation results of Benchmark functions of $G A, F A, D E$ and $A D E$.

\begin{tabular}{|c|c|c|c|c|c|}
\hline \multicolumn{6}{|c|}{ Dimension $=2$, population $=50$, iterations $=1000$} \\
\hline Function & Measure & GA & FA & $\mathrm{DE}$ & $\mathrm{ADE}$ \\
\hline$\overline{\text { Akely }}$ & minimum & 0.0263 & 1.2898 & $8.32 \mathrm{E}-07$ & $3.64 \mathrm{E}-07$ \\
\hline$(-4,5)$ & mean & 0.0624 & 1.4871 & $6.17 \mathrm{E}-06$ & $2.04 \mathrm{E}-06$ \\
\hline Global $\min =0$ & std. & 0.0409 & 0.1476 & $9.18 \mathrm{E}-06$ & $1.32 \mathrm{E}-06$ \\
\hline Powell & minimum & 0.0011 & $5.89 \mathrm{E}-06$ & $3.34 \mathrm{E}-04$ & 7.44E-05 \\
\hline$(-4,5)$ & mean & 0.0091 & 0.0127 & 0.003 & 0.0015 \\
\hline Global $\min =0$ & std. & 0.0058 & 0.0315 & 0.0032 & 0.0014 \\
\hline Beale & minimum & 0.0263 & $1.78 \mathrm{E}-07$ & $1.01 \mathrm{E}-07$ & $4.75 \mathrm{E}-08$ \\
\hline$(-4.5,4.5)$ & mean & 0.2321 & $1.14 \mathrm{E}-04$ & $9.23 \mathrm{E}-06$ & $2.37 \mathrm{E}-05$ \\
\hline Global $\min =0$ & std. & 0.3739 & $1.48 \mathrm{E}-04$ & $1.53 \mathrm{E}-05$ & $2.71 \mathrm{E}-05$ \\
\hline$\overline{\text { Bird }}$ & minimum & -106.765 & -106.765 & -106.765 & -106.765 \\
\hline (-2pi, 2pi) & mean & -102.874 & -106.753 & -106.764 & -106.764 \\
\hline Global $\min =106.7$ & std. & 8.2024 & 0.0152 & $6.39 \mathrm{E}-04$ & $3.65 \mathrm{E}-04$ \\
\hline Bukin4 & minimum & $1.30 \mathrm{E}-02$ & $4.55 \mathrm{E}-05$ & $4.70 \mathrm{E}-05$ & $2.95 \mathrm{E}-05$ \\
\hline$(-15,5)$ & mean & 0.0035 & 0.0013 & 7.6657 & 2.2355 \\
\hline Global $\min =0$ & std. & 0.0086 & 7.1094 & 16.0819 & 2.8937 \\
\hline Chichinadze & minimum & -42.4972 & -42.9441 & -41.7684 & -42.9444 \\
\hline$(-30,30)$ & mean & -42.4972 & -42.9224 & -41.8289 & -42.8605 \\
\hline Global $\min =-43.31$ & std. & 0.1414 & 0.0256 & 0.1365 & 0.1512 \\
\hline crosslegtable & minimum & -0.093 & -0.0198 & -0.013 & $-4.56 \mathrm{E}-04$ \\
\hline$(-10,10)$ & mean & -0.0049 & -0.0146 & -0.6511 & $-4.69 \mathrm{E}-04$ \\
\hline Global $\min =-1$ & std. & 0.0035 & 5.997 & 5.3809 & $5.22 \mathrm{E}-05$ \\
\hline goldsteinprice & minimum & 3 & 3 & 3 & $3.00 \mathrm{E}+00$ \\
\hline$(-2,2)$ & mean & 3 & 3 & 3 & $3.00 \mathrm{E}+00$ \\
\hline Global $\min =3$ & std. & $2.92 \mathrm{E}-15$ & $4.15 \mathrm{E}-15$ & $1.24 \mathrm{E}-06$ & 4.93E-07 \\
\hline himmelblau & minimum & 0.0023 & $2.43 \mathrm{E}-05$ & $7.01 \mathrm{E}-07$ & $4.87 \mathrm{E}-07$ \\
\hline$(-5,5)$ & mean & 0.312 & 0.0042 & $3.37 \mathrm{E}-04$ & 8.28E-04 \\
\hline Global $\min =0$ & std. & 0.0015 & 0.0051 & 7.33E-04 & 0.0016 \\
\hline schweffel & minimum & -587.966 & -637.909 & -837.966 & -837.966 \\
\hline$(-500,500)$ & mean & -555.056 & -658.499 & -837.966 & -837.966 \\
\hline Global $\min =-837.9$ & std. & 112.3693 & 101.39 & $7.71 \mathrm{E}-08$ & $5.52 \mathrm{E}-08$ \\
\hline testtubeholder & minimum & -10.8723 & -10.8722 & -10.8723 & -10.8723 \\
\hline$(-10,10)$ & mean & -10.6449 & -10.8713 & -10.8718 & -10.8723 \\
\hline Global $\min =-10.87$ & std. & 0.203622 & $9.01 \mathrm{E}-04$ & 0.0017 & $6.79 \mathrm{E}-05$ \\
\hline$\overline{\text { zettl }}$ & minimum & -0.0038 & -0.0038 & -0.0038 & -0.0038 \\
\hline$(-5,5)$ & mean & -0.0038 & -0.0037 & -0.0038 & -0.0038 \\
\hline Global $\min =-0.0037$ & std. & 2.89E-09 & $7.61 \mathrm{E}-05$ & $5.44 \mathrm{E}-10$ & 5.13E-09 \\
\hline
\end{tabular}

\section{PERFORMANCE EVALUATION OF ADE ALGORITHM}

In this section some comparisons between the genetic algorithm, firefly algorithm, DE and ADE using twelve numerical benchmark functions is demonstrated. The benchmark functions chosen for validation of Adaptive ADE over other optimizations are Ackley, Powell, Beale, Bird, Bukin4, Chichinadze, Crosslegtable, Goldsteinprice, Himmelblau, Schweffel, Testtubeholder and Zettl (Yao et al., 1999). Initially the algorithm is validated by taking dimension $=2$, population $=50$, iterations $=1000$ and performance measuring parameters like minimum, mean and standard deviation are considered. Table 1 shows the 12 benchmark functions and its corresponding range and theoretical minimum value. All the algorithms independently run for 50 times and minimum value is the minimum of objective function values of 50 independent runs. Mean or average is the ratio of the sum of the minimum value obtained to that of the number of independent runs. The mean value near to zero indicates better performance of the algorithm. The word 'std' is the standard deviation, which is equal to the 
square root of variance; a standard deviation close to zero indicates better performance of the algorithm and close to more than one indicates worst performance of the algorithm. In this work, our objective is to find the global minimum. Hence, lower the 'minimum', 'mean' and 'std.', better is the algorithm. In this work, the tuning parameters of GA are: crossover probability $=70 \%$ and mutation probability $=$ $20 \%$ and $\alpha=0.01, \beta_{0}=1$ and $\gamma=1$ are tuning parameters for FA and tuning parameters for $\mathrm{DE}$ and ADE are control parameter $(F)=1$, Crossover Rate $(C R)=10$, and strategy $=2$. Form Table 1, for Ackely function ADE minimum value is $3.6358 \mathrm{e}-07$ which is $0.0263,1.2898,4.6842 \mathrm{e}-07$ less than the GA, FA and DE respectively. With this example we can conclude that $\mathrm{ADE}$ is better than other algorithms as its minimum value is near to the theoretical value. The performance of the ADE algorithm is better than the GA, FA and DE, but for some benchmark functions the performance $\mathrm{ADE}$ is not so differentiable. For the benchmark functions Bird, Chichinadze, Goldsteinprice, Schweffel, Testtubeholder and Zettl the performance of ADE almost all similar to GA, FA and DE. Table. 2 shows the performance of ADE against GA, FA and DE on five benchmark functions with no change in dimension and population, but the number of iterations is reduced to 100. Even with lesser number of iterations ADE out perform the GA, FA and DE.

Table 3, 4 and 5 shows the performance of ADE against GA, FA and DE with 50 population, 100 iterations and dimensions are 30,60 and 100 respectively on three benchmark functions. These tables show that even higher dimensions of the problem ADE performance better than the GA, FA and DE. It is concluded that $\mathrm{ADE}$ algorithm outperforms the other algorithms in low dimensional and high dimensional search space.

Table 2. Dimension $=2$, population $=50$, iterations $=1000$.

\begin{tabular}{llcccc}
\hline Function & Measure & GA & FA & DE & ADE \\
\hline Akely & minimum & 1.0536 & 0.0022 & $2.98 \mathrm{E}-04$ & $2.21 \mathrm{E}-04$ \\
$(-4,5)$ & mean & 1.0536 & 0.0196 & 0.0019 & $6.22 \mathrm{E}-04$ \\
Global min $=0$ & std. & 0 & 0.0113 & 0.0013 & $2.71 \mathrm{E}-04$ \\
\hline Powell & minimum & 11.5801 & 0.0041 & 0.0467 & 0.001 \\
$(-4,5)$ & mean & 11.5801 & 0.0216 & 0.2286 & 0.0355 \\
Global min $=0$ & std. & 0 & 0.0186 & 0.2714 & 0.0343 \\
\hline Beale & minimum & 0.0077 & $4.62 \mathrm{E}-05$ & $3.88 \mathrm{E}-05$ & $2.24 \mathrm{E}-05$ \\
$(-4.5,4.5)$ & mean & 0.0077 & 0.0764 & 0.0019 & 0.0014 \\
Global min $=0$ & std. & 0 & 0.2409 & 0.0016 & 0.0015 \\
\hline Chichinadze & minimum & -42.9244 & -42.9415 & -42.9434 & -42.9443 \\
$(-30,30)$ & mean & -42.5205 & -42.8421 & -42.7111 & -42.8863 \\
Global min $=-43.31$ & std. & 0.1537 & 0.1823 & 0.2137 & 0.1378 \\
\hline testtubeholder & minimum & -10.8719 & -10.8717 & -10.8718 & -10.8723 \\
$(-10,10)$ & mean & -10.7198 & -10.8657 & -10.8635 & -10.8676 \\
Global min $=-10.87$ & std. & 0.2083 & 0.0087 & 0.0092 & 0.0061 \\
\hline
\end{tabular}

Table 3. Dimension $=30$, population $=50$, iterations $=100$.

\begin{tabular}{llcccc}
\hline Function & Measure & GA & FA & DE & ADE \\
\hline Akely & minimum & 4.4264 & 1.4553 & 7.6440 & 7.4363 \\
$(-4,5)$ & mean & 5.2181 & 1.7013 & 8.0370 & 7.8702 \\
Global min $=0$ & std. & 0.4574 & 0.1730 & 0.2483 & 0.2587 \\
\hline Exponential & minimum & 1.7131 & 1.4553 & 0.6440 & 0.4363 \\
$(-4,5)$ & mean & 0.5308 & 1.7013 & 0.0370 & 0.8702 \\
Global $\min =0$ & std. & 0.1182 & 0.1730 & 0.2483 & 0.2587 \\
\hline Brown & minimum & 64.2397 & 21.0185 & 7.2463 & 6.4224 \\
$(-4,5)$ & mean & 428.1775 & 21.5115 & 7.4442 & 5.5335 \\
Global $\min =0$ & std. & 847.7448 & 20.2839 & 2.0540 & 4.0634 \\
\hline
\end{tabular}


Table 4. Dimension $=60$, population $=50$, iterations $=100$.

\begin{tabular}{llcccc}
\hline Function & Measure & GA & FA & DE & ADE \\
\hline Akely & minimum & 4.4264 & 1.4553 & 7.644 & 3.4363 \\
$(-4,5)$ & mean & 5.2181 & 1.7013 & 8.037 & 3.8702 \\
Global min $=0$ & std. & 0.4574 & 0.173 & 0.2483 & 0.2587 \\
\hline Exponential & minimum & -0.4046 & -0.8453 & 3.3118 & 3.1224 \\
$(-4,5)$ & mean & -0.2614 & -0.7983 & 3.5443 & 3.5335 \\
Global $\min =0$ & std. & 0.0825 & 0.0329 & 0.1051 & 0.0634 \\
\hline Brown & minimum & 186.1389 & 59.5164 & 36.601 & 34.232 \\
$(-4,5)$ & mean & 985.7908 & 60.5707 & 47.61 & 35.344 \\
Global $\min =0$ & std. & $1.49 \mathrm{E}+03$ & 70.7381 & 14.919 & 13.121 \\
\hline
\end{tabular}

Table 5. Dimension $=100$, population $=50$, iterations $=100$.

\begin{tabular}{llcccc}
\hline Function & Measure & GA & FA & DE & ADE \\
\hline Akely & minimum & 15.2839 & 12.6322 & 8.9616 & 7.0844 \\
$(-4,5)$ & mean & 15.7639 & 12.7814 & 9.2831 & 7.1948 \\
Global min $=0$ & std. & 0.4142 & 0.0741 & 0.1338 & 0.0876 \\
\hline Exponential & minimum & -0.1214 & -0.6046 & 3.5954 & 3.4771 \\
$(-4,5)$ & mean & -0.0698 & -0.5453 & 3.6534 & 3.6021 \\
Global min $=0$ & std. & 0.0342 & 0.0482 & 0.0355 & 0.0711 \\
\hline Brown & minimum & $1.36 \mathrm{E}+03$ & 144.5975 & 39.05 & 11.893 \\
$(-4,5)$ & mean & $5.48 \mathrm{E}+07$ & 161.4384 & 40.089 & 13.021 \\
Global min $=0$ & std. & $1.73 \mathrm{E}+08$ & 123.4041 & 52.115 & 10.711 \\
\hline
\end{tabular}

The simulation results of six benchmark functions, i.e, Akely, Bukin4, Schweffel, Himmelbalu, Crosslegtable, and Zettle are shown in Fig. 7. These graphs are drawn between the number of iterations and the corresponding objective function value with 100 function evaluations. From these Figs., it is observed that ADE time of convergence is better to GA, FA and DE. Form Fig. 7a, one can compare perfor- mances of all four algorithms. Note that six figures (Figs. 7a-7e) are displayed for six benchmark functions separately. From Fig. 7, it is seen that our proposed algorithm ADE outperforms all other algorithms. So the proposed ADE is further used for codebook design in cascaded pattern search. The flow chart of proposed hybrid pattern search and ADE approach is shown in Fig. 8.

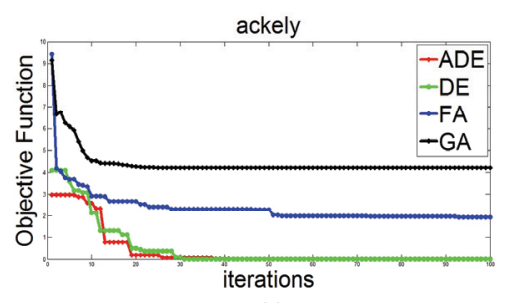

(a)

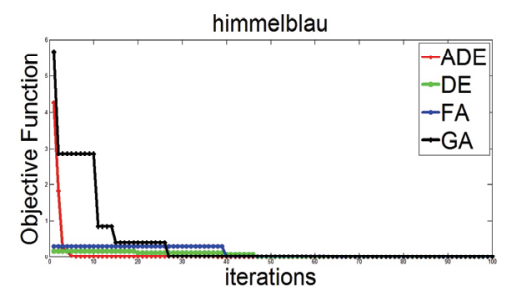

(d)

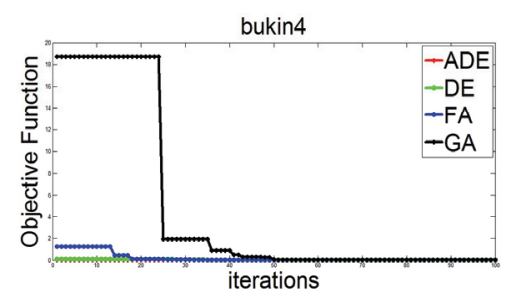

(b)

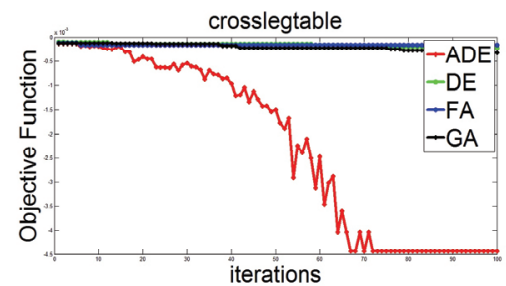

(e)

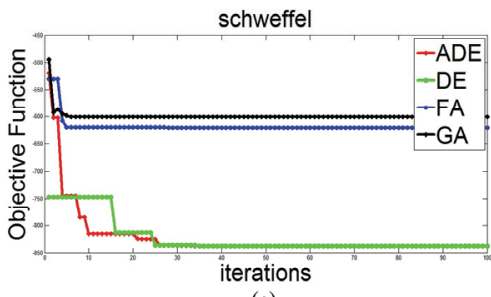

(c)

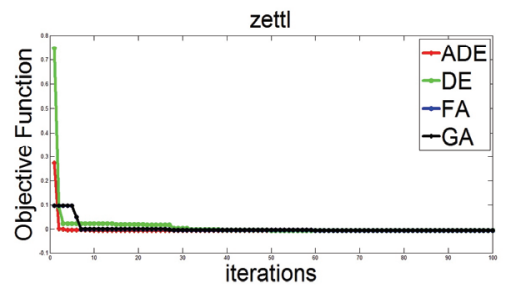

(f)

Fig. 7. Performance of proposed $A D E, D E, G A$ and $F A$ for $P=50$ and $D=30$. 


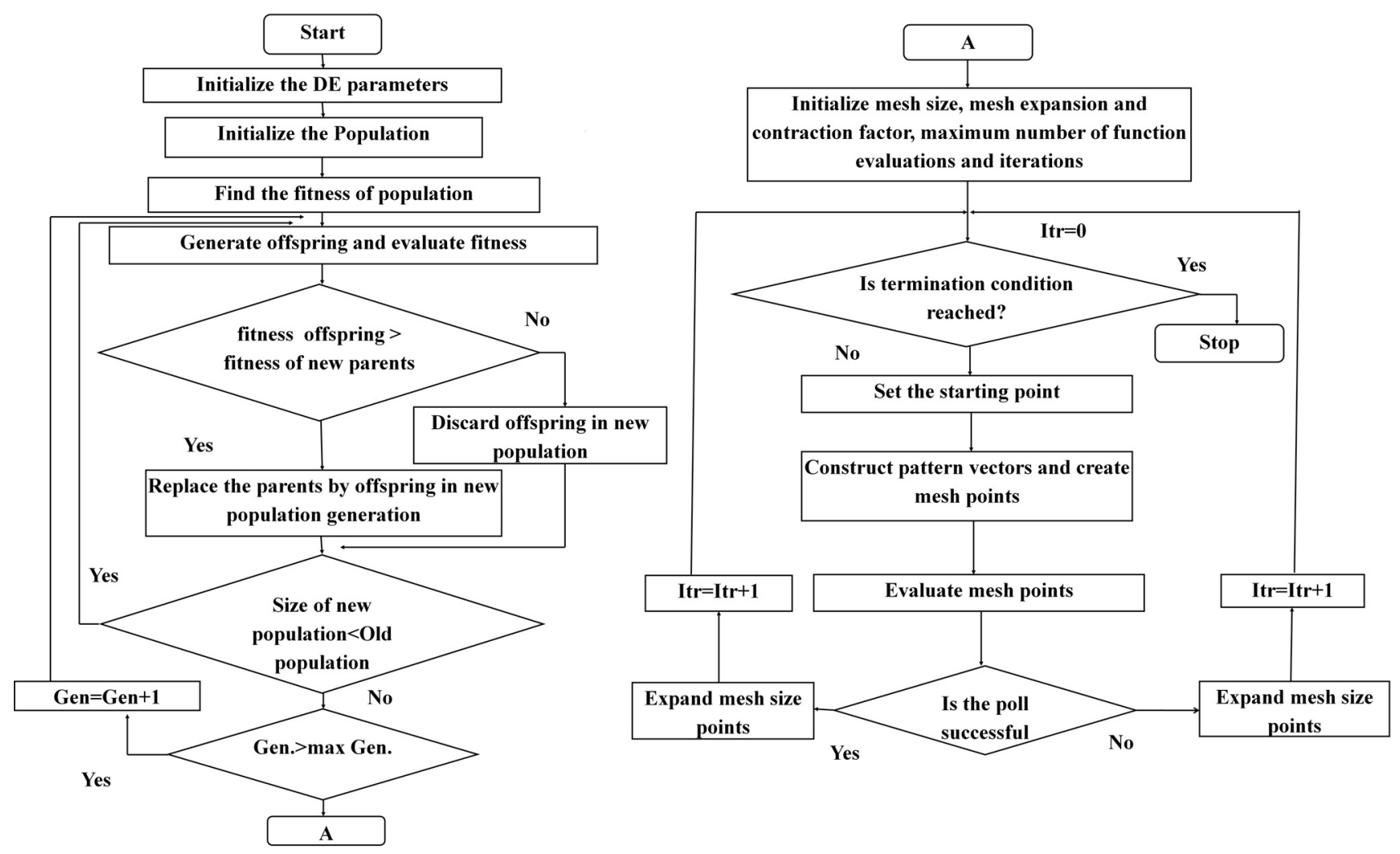

Fig. 8. Flowchart of proposed hADE-PS.

\section{RESULTS}

For the validation of the proposed hybrid ADE-PS method, four standard SAR images from the image database of compression Sandia National Laboratories 'flight line' and 'baseball diamond' and all the images are grayscale of size 256 by 256 stored in .jpg format are considered. The hybrid ADE-PS and other algorithms, i.e., K-Means, DE and ADE have been implemented in Matlab 7.9.0 (R2009b) on HP Laptop, Intel Core i5 processor 4GB RAM and 32 bit operating system. All the optimization algorithms acquired the K-Means codebook as the preliminary codebook or one of the population/solution. The parameters used for comparison of proposed hADE-PS algorithm with others are bitrate/bits per pixel, PSNR, Mean Square Error (MSE), SSIM, fitness function, compression ratio and memory size of output image.

Bitrate/Bits Per Pixel (BPP) \& Compression Ratio: Bitrate is the indication of number bits used to represent the vector quantized image, In this work two kinds of bitrates and compression ratio are defined; one before the run-length followed huffman coding and another after run-length followed Huffman coding. The formula for the bitrate and compression ratio of the first one $(B P P \& C R)$ and second one $\left(B P P_{\text {code }} \&\right.$ $C R_{\text {code }}$ ) is given in Eq. 9 and Eq. 10 respectively.

$$
\mathrm{BPP}=\frac{\log _{2} N_{c}}{k} \quad \mathrm{CR}=\frac{8 \times k}{N_{c}},
$$

where $N_{c}$ is codebook size and $\mathrm{k}$ is non-overlapping image block size $(4 * 4)$

$$
\begin{aligned}
& \text { bpp }=\frac{\text { total number of bits transmitted }}{\text { size of image }} \\
& C R_{\text {code }}=\frac{\text { size of image } * 8}{\text { total number of bits transmitted }}
\end{aligned}
$$

Peak Signal to Noise Ratio (PSNR): It's a measure of reconstructed/decompressed image quality which is given in Eq. 11

$$
P S N R=10 \times \log _{10}\left(\frac{255^{2}}{M S E}\right)(\mathrm{dB})
$$

where Mean Square Error (MSE) which is given in Eq. 12

$$
M S E=\frac{1}{M \times N} \sum_{I}^{M} \sum_{J}^{N}\{f(\mathrm{I}, \mathrm{J})-\bar{f}(\mathrm{I}, \mathrm{J})\}^{2},
$$

where $M \times N$ is the size of the image, $I$ and $J$ represents the pixel value of original and decompressed images. In our experiment we have taken $N=M$ a square image. $f(I, J)$ is an original image and $\bar{f}(I, J)$ reconstructed image of size 256 by 256 . 
Structural Similarity Index Measure (SSIM): It evaluates the visual similarity between the original image and the reconstructed image. High-quality image is one whose structure closely matches that of the original input image. The structural similarity index is calculated between original input image and reconstructed image given in Eq. 13.

$$
\operatorname{SSIM}=\frac{\left(2 \mu_{I} \mu_{\widetilde{I}}+C_{1}\right)\left(2 \sigma_{I \widetilde{I}}+C_{2}\right)}{\left(\mu_{I}^{2}+\mu_{\widetilde{I}}^{2}-C_{1}\right)\left(\sigma_{I}^{2}+\sigma_{\widetilde{I}}^{2}-C_{2}\right)},
$$

where $\mu_{I}$ and $\mu_{I}$ are the mean value of the original image $I$ and reconstructed image $\tilde{I}, \sigma_{I}$ and $\sigma_{\tilde{I}}$ are the standard deviation of original image $I$ and reconstructed image $\tilde{I}, \sigma_{I I}$ is the cross-correlation and $C_{l} \&$ $C_{2}$ are constants are equal to 0.065 .

$$
\text { Mean value }=\mu_{I}=\frac{1}{N} \sum_{i=1}^{N} I_{i} .
$$

Standard deviation $=$

$$
\sigma_{\tilde{I}}=\frac{1}{N-1} \sum_{i=1}^{N}\left(\mathrm{I}_{i}-\mu_{I}\right)\left(\tilde{\mathrm{I}}_{i}-\mu_{\tilde{I}}\right) .
$$

\section{DISCUSSIONS}

The proposed hADE-PS based vector quantization is compared with the standard K-Means, Differential Evolution and Adaptive Differential evolution. The proposed method is evaluated with a codebook size of $8,16,32,64,128,256$ and 512 and each algorithm runs five times. It is observed that PSNR value is increased with increment in codebook size. The same parameters are used for all experiments (for consistency).

Fig. 9 to Fig. 12 shows the graph between BPP to PSNR and bitrate to the fitness function. In the proposed work fitness function is the ratio of sum of the Euclidean distance between the input image and codewords (distortion) to size of the input image. So motto is to design or optimize codebook which minimizes distortion. From the figures, it can be observed that the peak signal to noise ratio of hADEPS is superior to the K-Means, DE and ADE. The PSNR value of hADE-PS is around 0.1 to 0.3 higher than the K-Means, DE and ADE because of its exploration and exploitation behavior. This 0.1 to 0.3 is not a huge difference, but its reflection on MSE between the input image and the reconstructed image is very huge (around 10 times lesser). This is specified in Fig. 13 to Fig. 16, where a difference of 0.1 in PSNR between ADE and hADE-PS is equal to the difference in MSE of around 283099 for flight line image. Fig. 13 to Fig. 16 shows the bar chart of MSE of four methods for four images and observed MSE is decreasing with the increment in codebook size and MSE of hADE-PS is considerable smaller than the KMeans, DE and ADE.

From Table 6 and 7, it is observed that the proposed hADE-PS method achieved lesser bits per pixel at an average of $0.77 \%$ and $0.56 \%$ as compared to $\mathrm{DE}$ and $\mathrm{ADE}$ and higher at an average of $2.77 \%$ as compared to K-Means. hADE-PS achieved higher compression ratio at an average of $5.6 \%$ and $4.4 \%$ compared to $\mathrm{DE}$ and $\mathrm{ADE}$ and lesser at an average of $1.24 \%$ compared to K-Means for Baseball SAR image. Whereas hADE-PS method achieved higher SSIM for Baseball SAR image at an average of $0.52 \%$ compared to K-Means and lesser at an average of $1.50 \%$ and $1.36 \%$ compared to DE and ADE respectively.
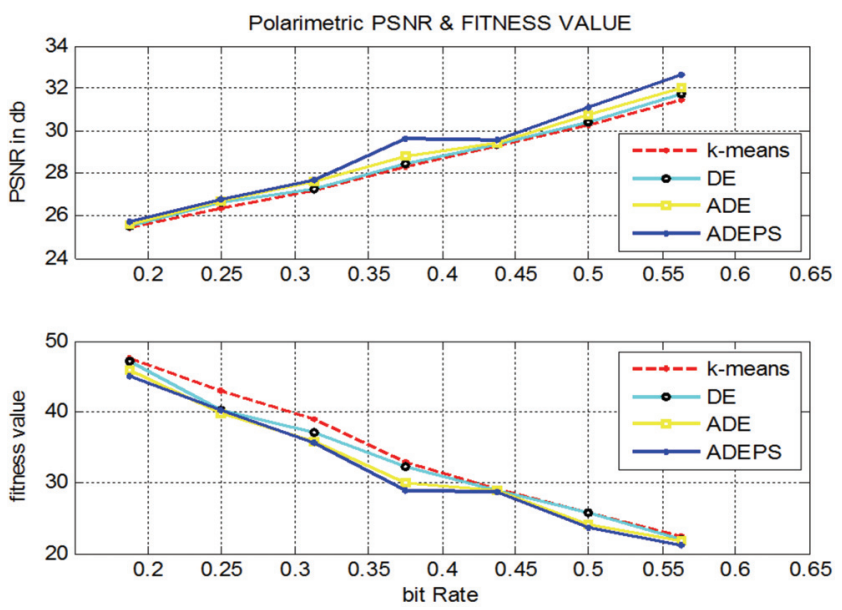

Fig. 9. PSNR \& fitness function of Polarimetric SAR image.
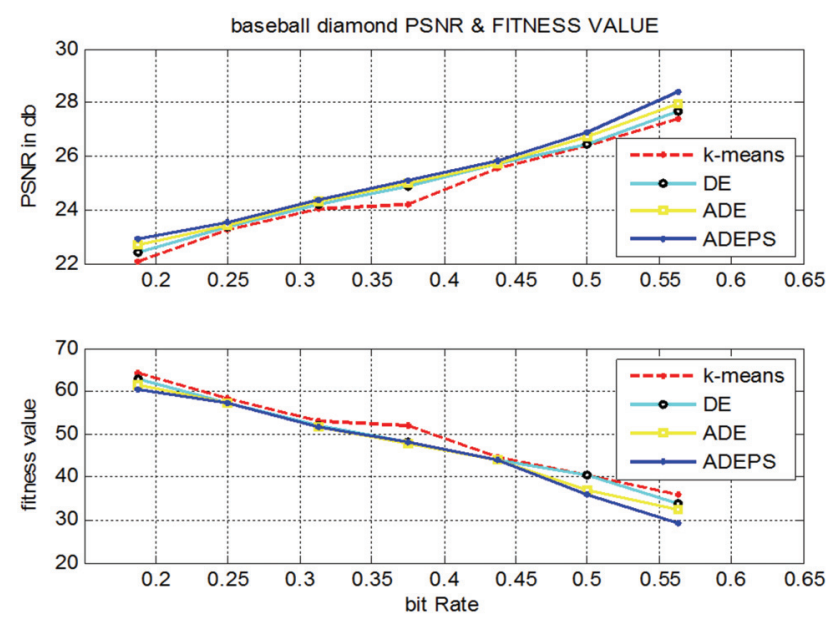

Fig. 10. PSNR \& fitness function of BaseBall Diamond SAR image. 

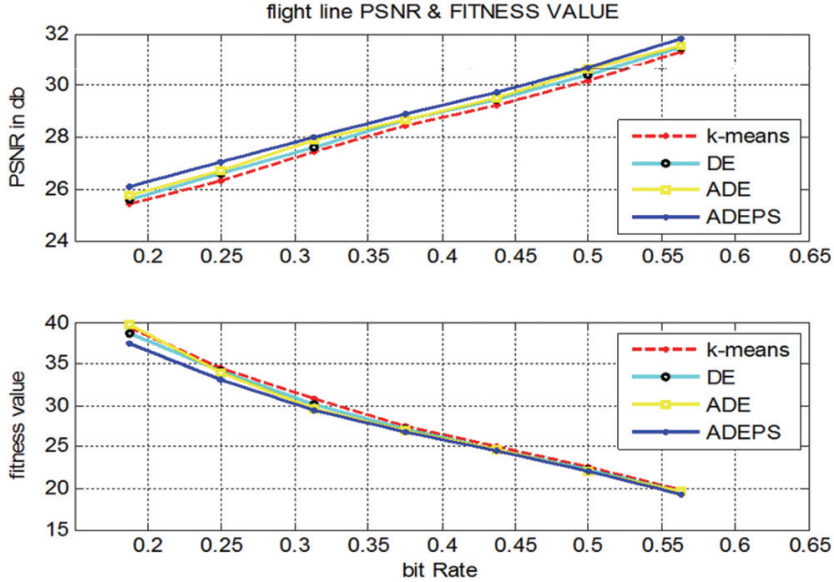

Fig. 11. PSNR \& fitness function of Flight Line SAR image.
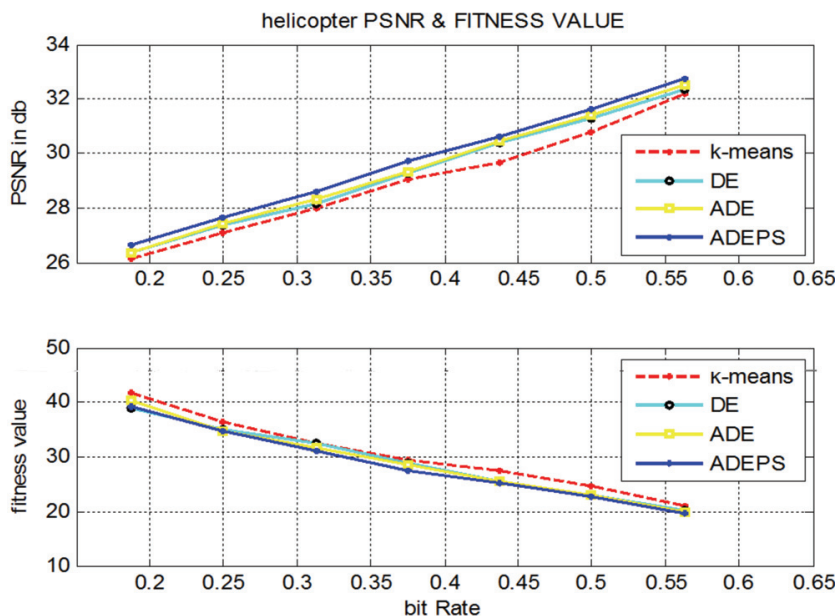

Fig. 12. PSNR \& fitness function of Flight Line SAR image.

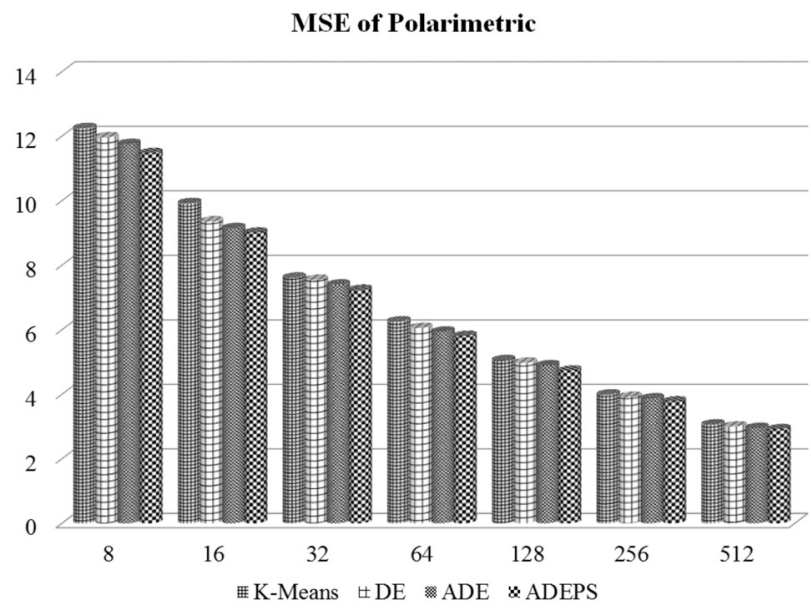

Fig. 13. Polarimetric image MSE with a codebook size of 8, 16, 32, 64, 128, 256 and 512 .

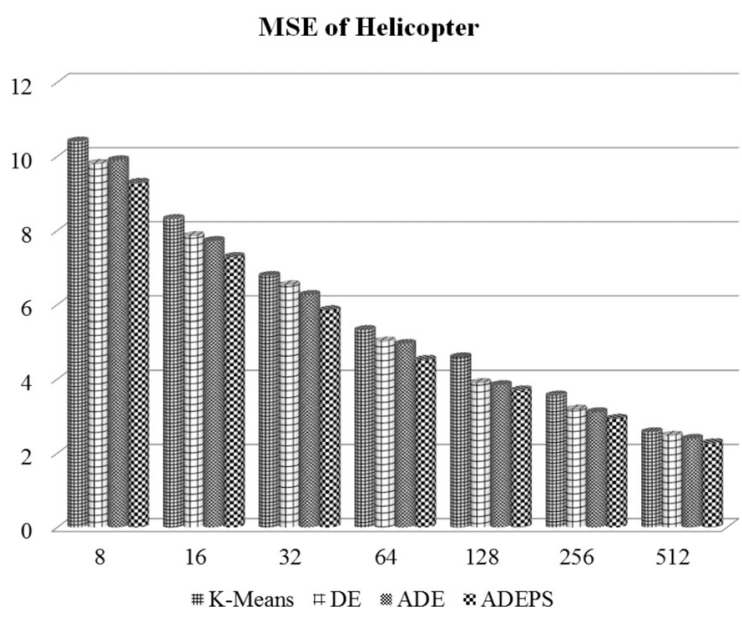

Fig. 14. Helicopter image MSE with a codebook size of $8,16,32,64,128,256$ and 512.

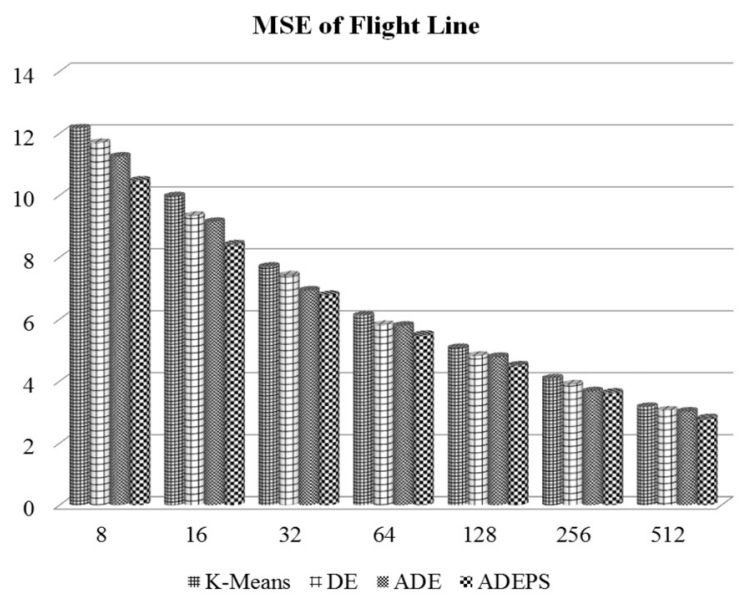

Fig. 15. Flight Line image MSE with a codebook size of $8,16,32,64,128,256$ and 512.

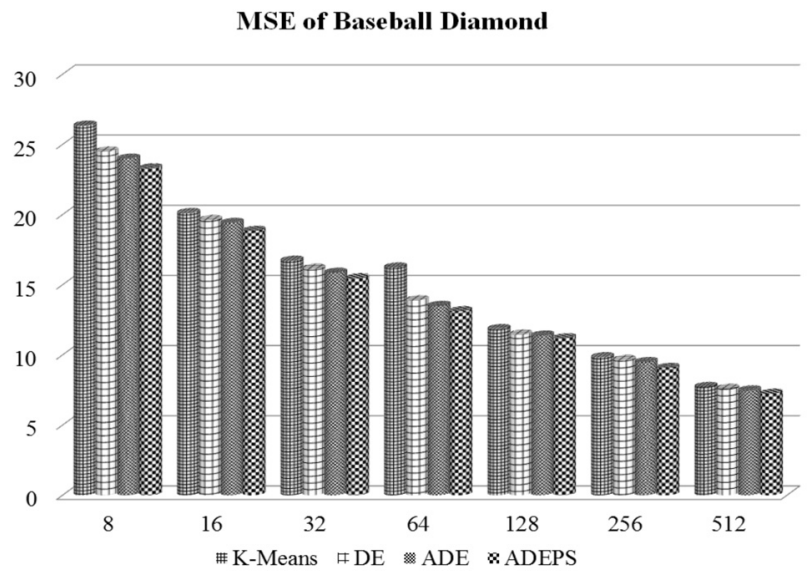

Fig. 16. Baseball Diamond image MSE with a code book size of 8, 16, 32, 64, 128, 256 and 512. 
From Table 8 and 9, it is observed that the proposed hADE-PS method achieved lesser bits per pixel at an average of $0.27 \%$ and $1.15 \%$ compared to K-Means and $\mathrm{DE}$ and higher at an average of $0.41 \%$ compared to $\mathrm{ADE}$ and achieved higher compression ratio at an average of $0.8 \%$ and $2.6 \%$ compared to K-Means and $\mathrm{DE}$ and lesser at an average of $7.09 \%$ compared to ADE for Flight line SAR image. Whereas hADE-PS method achieved higher SSIM at an average of $1.02 \%, 0.03 \%$ and $1.28 \%$ compared to K-Means, DE and ADE respectively for Flight line SAR image.

From Table. 10 and 11, it is observed that the proposed hADE-PS method achieved lesser bits per Pixelat an average of $1.65 \%, 0.80 \%$ and $0.90 \%$ with compare to K-Means, DE and ADE respectively, and hADE-PS method achieved higher compression ratio at an average of $7.57 \%, 5.29 \%$ and $6.06 \%$ with compare to K-Means, DE and ADE respectively for Helicopter SAR image. Whereas hADE-PS method achieved higher SSIM at an average of $0.13 \%$, and $0.29 \%$ compared to K-Means and DE respectively, and lesser at an average of $0.64 \%$ compared to ADE.

From Table. 12 and 13, it is observed that the proposed hADE-PS method achieved higher bits per Pixelat an average of $1.17 \%$ and $0.3 \%$ compared to $\mathrm{K}-\mathrm{Means}$ and DE and no difference compared to ADE and hADE-PS method achieved higher compression ratio at an average of $0.33 \%$ compared to ADE and lesser at an average of $4.09 \%$ and $1.88 \%$ compared to K-Means and DE Polarimetric SAR image. Whereas hADE-PS method achieved higher SSIM at an average of $1.06 \%, 0.56 \%$ and $0.41 \%$ compared to K-Means, DE and ADE respectively. From Table 6-13 it is observed that a lower and lower value of minimum value of output images and a higher and higher value of maximum value of output image shows the better PSNR and SSIM.

Table 6. The bits per pixel (BPP), Compression ratio (CR), Structural similarity index (SSIM), Number of bits for codebook (bcodebook) and index (bindex), min \& max of output image and the number of bytes (bout) of Baseball images by using the four different algorithms with different codebook size (NC).

\begin{tabular}{llccccccc}
\hline $\mathrm{N}_{\mathrm{C}}$ & Technique & $\mathrm{BPP}_{\text {code }}$ & $\mathrm{CR}_{\text {code }}$ & SSIM & $\mathrm{b}_{\text {codebook }}$ & $\mathrm{b}_{\text {index }}$ & $\min \&$ max & $\mathrm{b}_{\text {out }}$ \\
\hline 8 & K-Means & 0.145142 & 55.11859 & 0.315150 & 680 & 8832 & 25,157 & 1189 \\
& DE & 0.159668 & 50.10398 & 0.342557 & 728 & 9736 & 31175 & 1308 \\
& ADE & 0.153564 & 52.09539 & 0.324821 & 688 & 9376 & 34,168 & 1258 \\
& ADEPS & 0.143555 & 55.72789 & 0.308223 & 728 & 8680 & 37,179 & 1176 \\
\hline 16 & K-Means & 0.222778 & 35.91014 & 0.431154 & 1600 & 13000 & 26,179 & 1825 \\
& DE & 0.215332 & 37.15193 & 0.416324 & 1616 & 12496 & 25,179 & 1764 \\
& ADE & 0.217651 & 36.75603 & 0.411986 & 1624 & 12640 & 25,178 & 1783 \\
& ADEPS & 0.201172 & 39.76699 & 0.405823 & 1640 & 11544 & 27,180 & 1648 \\
\hline 32 & K-Means & 0.299316 & 26.72757 & 0.493829 & 3320 & 16296 & 25,181 & 2452 \\
& DE & 0.307373 & 26.02701 & 0.496363 & 3408 & 16736 & 19,186 & 2518 \\
& ADE & 0.304443 & 26.27747 & 0.499020 & 3384 & 16568 & 23,184 & 2494 \\
& ADEPS & 0.297607 & 26.88105 & 0.486835 & 3352 & 16152 & 21,191 & 2438 \\
\hline 64 & K-Means & 0.311768 & 25.66014 & 0.499794 & 3376 & 17056 & 24,187 & 2554 \\
& DE & 0.430664 & 18.57596 & 0.566458 & 6880 & 21344 & 19,196 & 3528 \\
& ADE & 0.429565 & 18.62347 & 0.574566 & 6896 & 21256 & 18,198 & 3519 \\
& ADEPS & 0.420776 & 19.01247 & 0.565834 & 7040 & 20536 & 9,250 & 3447 \\
\hline 128 & K-Means & 0.613525 & 13.03940 & 0.637223 & 13800 & 26408 & 16,205 & 5026 \\
& DE & 0.611938 & 13.07321 & 0.637296 & 13872 & 26232 & 16,218 & 5013 \\
& ADE & 0.609863 & 13.11769 & 0.639557 & 13856 & 26112 & 16,212 & 4996 \\
& ADEPS & 0.607056 & 13.17836 & 0.635659 & 14048 & 25736 & 8,222 & 4973 \\
\hline 256 & K-Means & 0.884277 & 9.046935 & 0.704460 & 27336 & 30616 & 16,222 & 7244 \\
& DE & 0.890137 & 8.987383 & 0.702164 & 27712 & 30624 & 12,231 & 7292 \\
& ADE & 0.890747 & 8.981225 & 0.703864 & 27680 & 30696 & 16,223 & 7297 \\
& ADEPS & 0.898804 & 8.900720 & 0.704363 & 28208 & 30696 & 14,243 & 7363 \\
\hline 512 & K-Means & 1.379639 & 5.798620 & 0.767407 & 55264 & 35152 & 7,242 & 11302 \\
& DE & 1.382202 & 5.787865 & 0.766288 & 55464 & 35120 & 4,250 & 11323 \\
& ADE & 1.383301 & 5.783269 & 0.768358 & 55552 & 35104 & 10,237 & 11332 \\
& ADEPS & 1.397705 & 5.723668 & 0.762571 & 56576 & 35024 & 9,243 & 11450 \\
\hline
\end{tabular}


Table 7. Average BPP code, $C R_{\text {code, }}$ SSIM and \% difference against ADEPS of Baseball SAR image.

\begin{tabular}{lcccccc}
\hline & BPP $_{\text {code }}$ & $\begin{array}{c}\text { \% difference } \\
\text { against ADEPS }\end{array}$ & CR $_{\text {code }}$ & $\begin{array}{c}\text { \% difference } \\
\text { against ADEPS }\end{array}$ & SSIM & $\begin{array}{c}\text { \% difference } \\
\text { against ADEPS }\end{array}$ \\
\hline K-Means & 0.550921 & 2.778876 & 24.47163 & -1.24728 & 0.549860 & 0.52428 \\
DE & 0.571045 & -0.77241 & 22.81533 & 5.605383 & 0.561064 & -1.50265 \\
ADE & 0.569876 & -0.56612 & 23.09065 & 4.466292 & 0.560310 & -1.36624 \\
ADEPS & 0.566668 & & 24.17016 & & 0.552758 & \\
\hline
\end{tabular}

Table 8. The bits per pixel (BPP), Compression ratio (CR), Structural similarity index (SSIM), Number of bits for codebook (bcodebook) and index (bindex), min \& max of output image and the number of bytes (bout) of Flight line images by using the four different algorithms with different codebook size (NC).

\begin{tabular}{llccccccc}
\hline $\mathrm{N}_{\mathrm{C}}$ & Technique & $\mathrm{BPP}_{\text {code }}$ & $\mathrm{CR}_{\text {code }}$ & $\mathrm{SSIM}$ & $\mathrm{b}_{\text {codebook }}$ & $\mathrm{b}_{\text {index }}$ & $\min \& \max$ & $\mathrm{b}_{\text {out }}$ \\
\hline 8 & K-Means & 0.114014 & 70.16702 & 0.220490 & 600 & 6872 & 10,102 & 934 \\
& DE & 0.116699 & 68.55230 & 0.225755 & 560 & 7088 & 15,113 & 956 \\
& ADE & 0.097290 & 82.22836 & 0.198176 & 560 & 5816 & 18,116 & 797 \\
& ADEPS & 0.112427 & 71.15744 & 0.226754 & 600 & 6768 & 16,155 & 921 \\
\hline 16 & K-Means & 0.184326 & 43.40132 & 0.304027 & 1400 & 10680 & 7,116 & 1510 \\
& DE & 0.188843 & 42.36328 & 0.312903 & 1352 & 11024 & 8,131 & 1547 \\
& ADE & 0.168945 & 47.35260 & 0.290382 & 1376 & 9696 & 6,160 & 1384 \\
& ADEPS & 0.176636 & 45.29095 & 0.294902 & 1408 & 10168 & 9,180 & 1447 \\
\hline 32 & K-Means & 0.260498 & 30.71040 & 0.366904 & 2944 & 14128 & 7,147 & 2134 \\
& DE & 0.264404 & 30.25669 & 0.366673 & 2984 & 14344 & 6,160 & 2166 \\
& ADE & 0.258911 & 30.89863 & 0.371584 & 3016 & 13952 & 6,161 & 2121 \\
& ADEPS & 0.267822 & 29.87056 & 0.366819 & 3088 & 14464 & 6,184 & 2194 \\
\hline 64 & K-Means & 0.386108 & 20.71957 & 0.444107 & 6200 & 19104 & 4,174 & 3163 \\
& DE & 0.383301 & 20.87134 & 0.439294 & 6360 & 18760 & 3,185 & 3140 \\
& ADE & 0.389404 & 20.54420 & 0.445250 & 6288 & 19232 & 5,180 & 3190 \\
& ADEPS & 0.381470 & 20.97152 & 0.434910 & 6456 & 18544 & 6,189 & 3125 \\
\hline 128 & K-Means & 0.559692 & 14.29357 & 0.515133 & 12544 & 24136 & 3,187 & 4585 \\
& DE & 0.571899 & 13.98847 & 0.527041 & 12808 & 24672 & 3,197 & 4685 \\
& ADE & 0.571167 & 14.00641 & 0.522511 & 13064 & 24368 & 2,187 & 4679 \\
& ADEPS & 0.574829 & 13.91718 & 0.521636 & 13400 & 24272 & 4,196 & 4709 \\
\hline 256 & K-Means & 0.855713 & 9.348930 & 0.605669 & 26096 & 29984 & 2,202 & 7010 \\
& DE & 0.866699 & 9.230423 & 0.614450 & 26656 & 30144 & 2,215 & 7100 \\
& ADE & 0.859497 & 9.307769 & 0.607158 & 26720 & 29608 & 3,227 & 7041 \\
& ADEPS & 0.861938 & 9.281405 & 0.604545 & 26800 & 29688 & 2,235 & 7061 \\
\hline 512 & K-Means & 1.338623 & 5.976290 & 0.694850 & 52808 & 34920 & 0,202 & 10966 \\
& DE & 1.344360 & 5.9507850 & 0.692779 & 53296 & 34808 & 0,211 & 11013 \\
& ADE & 1.344238 & 5.9513260 & 0.694665 & 53248 & 34848 & 1,211 & 11012 \\
& ADEPS & 1.362305 & 5.8724010 & 0.693082 & 54416 & 34864 & 0,234 & 11160 \\
\hline
\end{tabular}

Table 9. Average BPP code, $C R_{\text {code }}$ SSIM and \% difference against ADEPS of Flight line SAR image.

\begin{tabular}{lcccccc}
\hline & $\mathrm{BPP}_{\text {code }}$ & $\begin{array}{c}\text { \% difference } \\
\text { against ADEPS }\end{array}$ & $\mathrm{CR}_{\text {code }}$ & $\begin{array}{c}\text { \% difference } \\
\text { against ADEPS }\end{array}$ & SSIM & $\begin{array}{c}\text { \% difference } \\
\text { against ADEPS }\end{array}$ \\
\hline K-Means & 0.450169 & -0.27152 & 27.80244 & 0.888362 & 0.528425 & 1.028810 \\
DE & 0.454128 & -1.15336 & 27.31618 & 2.621807 & 0.533744 & 0.032589 \\
ADE & 0.447104 & 0.411182 & 30.04133 & -7.09295 & 0.527065 & 1.283530 \\
ADEPS & 0.448950 & & 28.05164 & & 0.533918 & \\
\hline
\end{tabular}


Table 10. The bits per pixel (BPP), Compression ratio (CR), Structural similarity index (SSIM), Number of bits for codebook $\left(b_{\text {codebook }}\right)$ and index $\left(b_{\text {index }}\right), \min \&$ max of output image and the number of bytes $\left(b_{\text {out }}\right)$ of Helicopter images by using the four different algorithms with different codebook size $\left(N_{C}\right)$.

\begin{tabular}{llccccccc}
\hline $\mathrm{N}_{\mathrm{C}}$ & Technique & $\mathrm{BPP}_{\text {code }}$ & $\mathrm{CR}_{\text {code }}$ & SSIM & $\mathrm{b}_{\text {codebook }}$ & $\mathrm{b}_{\text {index }}$ & min \& max & $\mathrm{b}_{\text {out }}$ \\
\hline 8 & K-Means & 0.168213 & 47.55878 & 0.331316 & 688 & 10336 & 11,78 & 1,378 \\
& DE & 0.166015 & 48.18800 & 0.320182 & 688 & 10192 & 11,77 & 1360 \\
& ADE & 0.173462 & 46.11963 & 0.342659 & 672 & 10696 & 6,85 & 1,421 \\
& ADEPS & 0.138306 & 57.84289 & 0.317535 & 640 & 8424 & 11,78 & 1,133 \\
\hline 16 & K-Means & 0.235474 & 33.97408 & 0.407295 & 1416 & 14016 & 10,94 & 1,929 \\
& DE & 0.220703 & 36.24779 & 0.399017 & 1456 & 13008 & 5,83 & 1808 \\
& ADE & 0.216919 & 36.88014 & 0.405095 & 1424 & 12792 & 3,78 & 1,777 \\
& ADEPS & 0.227783 & 35.12111 & 0.409797 & 1544 & 13384 & 5,191 & 1,866 \\
\hline 32 & K-Means & 0.318970 & 25.08075 & 0.476180 & 2984 & 17920 & 6,88 & 2613 \\
& DE & 0.312500 & 25.60000 & 0.472684 & 3048 & 17432 & 6,11 & 2560 \\
& ADE & 0.305298 & 26.20392 & 0.467926 & 3088 & 16920 & 7,80 & 2501 \\
& ADEPS & 0.315918 & 25.32303 & 0.479451 & 3096 & 17608 & 3,203 & 2588 \\
\hline 64 & K-Means & 0.428101 & 18.68720 & 0.541240 & 6096 & 21960 & 5,177 & 3507 \\
& DE & 0.423218 & 18.90280 & 0.532136 & 6240 & 21496 & 3,173 & 3467 \\
& ADE & 0.433105 & 18.47125 & 0.547188 & 6272 & 22112 & 3,197 & 3548 \\
& ADEPS & 0.409546 & 19.53383 & 0.529461 & 6344 & 20496 & 3,203 & 3355 \\
\hline 128 & K-Means & 0.601807 & 13.29331 & 0.606344 & 12680 & 26760 & 2,188 & 4930 \\
& DE & 0.592773 & 13.49588 & 0.612615 & 12568 & 26280 & 2,203 & 4856 \\
& ADE & 0.595093 & 13.44328 & 0.609848 & 12792 & 26208 & 1,203 & 4875 \\
& ADEPS & 0.597290 & 13.39383 & 0.611974 & 26136 & 26136 & 1,253 & 4893 \\
\hline 256 & K-Means & 0.858643 & 9.317032 & 0.673415 & 25072 & 31200 & 1,197 & 7034 \\
& DE & 0.862427 & 9.276150 & 0.684333 & 25528 & 30992 & 2,203 & 7065 \\
& ADE & 0.860962 & 9.291933 & 0.683863 & 25416 & 31008 & 1,203 & 7053 \\
& ADEPS & 0.860107 & 9.301164 & 0.683592 & 25656 & 30712 & 1,253 & 7046 \\
\hline 512 & K-Means & 1.318237 & 6.068710 & 0.744965 & 51048 & 35344 & 0,255 & 10799 \\
& DE & 1.318970 & 6.065340 & 0.753700 & 51152 & 35288 & 0,203 & 10805 \\
& ADE & 1.315918 & 6.079406 & 0.753683 & 50984 & 35256 & 0,253 & 10780 \\
& ADEPS & 1.316650 & 6.076024 & 0.754213 & 51144 & 35144 & 0,255 & 10786 \\
\hline
\end{tabular}

Table 11. Average BPP $P_{\text {code }}, C R_{\text {code }}, S S I M$ and \% difference against ADEPS of Helicopter SAR image.

\begin{tabular}{lcccccc}
\hline & $\mathrm{BPP}_{\text {code }}$ & $\begin{array}{c}\text { \% difference } \\
\text { against ADEPS }\end{array}$ & $\mathrm{CR}_{\text {code }}$ & $\begin{array}{c}\text { \% difference } \\
\text { against ADEPS }\end{array}$ & SSIM & $\begin{array}{c}\text { \% difference } \\
\text { against ADEPS }\end{array}$ \\
\hline K-Means & 0.561349 & -1.65162 & 21.99712 & 7.570621 & 0.540108 & 0.139038 \\
DE & 0.556658 & -0.8021 & 22.53942 & 5.291939 & 0.539238 & 0.299893 \\
ADE & 0.557251 & -0.90948 & 22.35565 & 6.064119 & 0.544323 & -0.64028 \\
ADEPS & 0.552228 & & 23.79884 & & 0.540860 & \\
\hline
\end{tabular}

Fig. 17 to Fig. 20 shows the reconstructed images of 'Baseball diamond' 'Flight line', 'Helicopter', and 'Polarimetric', with a codebook size of $64,16,8$ and 8 respectively for K-Means, DE, ADE and hADE-PS. These codebook size is selected only for the better explanation and visual proof of the proposed method. For the best explanation of stated method, the regions marked with the red pencil shows reconstructed image visible clearly with the proposed method. For flight line image the regions marked with red are smoother with hADE-PS, whereas with other methods these regions are blurred as shown in Fig. 18. Similarly for Baseball diamond, Helicopter and Polarimetric the marked red regions are smooth with hADE-PS compared to other K-Means, DE, ADE optimization techniques. 
Table 12. The bits per pixel (BPP), Compression ratio (CR), Structural similarity index (SSIM), Number of bits for codebook $\left(b_{\text {codebook }}\right)$ and index $\left(b_{\text {index }}\right)$, min \& max of output image and the number of bytes $\left(b_{\text {out }}\right)$ of Polarimetric images by using the four different algorithms with different codebook size $\left(N_{C}\right)$.

\begin{tabular}{llccccccc}
\hline $\mathrm{N}_{\mathrm{C}}$ & Technique & $\mathrm{BPP}_{\text {code }}$ & $\mathrm{CR}_{\text {code }}$ & SSIM & $\mathrm{b}_{\text {codebook }}$ & $\mathrm{b}_{\text {index }}$ & $\min \& \max$ & $\mathrm{b}_{\text {out }}$ \\
\hline 8 & K-Means & 0.153931 & 51.97145 & 0.383693 & 712 & 9376 & 27,122 & 1261 \\
& DE & 0.154907 & 51.64381 & 0.384900 & 704 & 9448 & 26,121 & 1269 \\
& ADE & 0.166870 & 47.94148 & 0.393032 & 720 & 10216 & 24,120 & 1367 \\
& ADEPS & 0.167725 & 47.69723 & 0.399023 & 704 & 10288 & 18,120 & 1374 \\
\hline 16 & K-Means & 0.220825 & 36.22775 & 0.473461 & 1560 & 12912 & 24,130 & 1809 \\
& DE & 0.236206 & 33.86873 & 0.488854 & 1520 & 13960 & 3,120 & 1935 \\
& ADE & 0.234009 & 34.18675 & 0.483875 & 1480 & 13856 & 5,124 & 1917 \\
& ADEPS & 0.231567 & 34.54718 & 0.492617 & 1536 & 13640 & 17,124 & 1897 \\
\hline 32 & K-Means & 0.318481 & 25.11920 & 0.564017 & 3184 & 17688 & 3,141 & 2609 \\
& DE & 0.321899 & 24.85248 & 0.568047 & 3104 & 17992 & 14,133 & 2637 \\
& ADE & 0.321655 & 24.87135 & 0.569604 & 3240 & 17840 & 9,127 & 2635 \\
& ADEPS & 0.315918 & 25.32303 & 0.564702 & 3216 & 17488 & 7,144 & 2588 \\
\hline 64 & K-Means & 0.424194 & 18.85928 & 0.631893 & 6320 & 21480 & 0,167 & 3475 \\
& DE & 0.432495 & 18.49732 & 0.635423 & 6544 & 21800 & 2,184 & 3543 \\
& ADE & 0.431763 & 18.52870 & 0.630889 & 6528 & 21768 & 2,147 & 3537 \\
& ADEPS & 0.433838 & 18.44007 & 0.639282 & 6688 & 21744 & 2,191 & 3554 \\
\hline 128 & K-Means & 0.598145 & 13.37469 & 0.699715 & 13104 & 26096 & 1,155 & 4900 \\
& DE & 0.601929 & 13.29061 & 0.699861 & 13376 & 26072 & 1,175 & 4931 \\
& ADE & 0.606079 & 13.19960 & 0.708086 & 13408 & 26312 & 1,162 & 4965 \\
& ADEPS & 0.601929 & 13.29061 & 0.701873 & 13488 & 25960 & 1,175 & 4931 \\
\hline 256 & K-Means & 0.872681 & 9.167156 & 0.759742 & 26576 & 30616 & 0,176 & 7149 \\
& DE & 0.870972 & 9.185144 & 0.757715 & 26680 & 30400 & 1,179 & 7135 \\
& ADE & 0.871582 & 9.178711 & 0.756357 & 26744 & 30376 & 1,191 & 7140 \\
& ADEPS & 0.874756 & 9.145409 & 0.762382 & 27016 & 30312 & 1,187 & 7166 \\
\hline 512 & K-Means & 1.349731 & 5.927105 & 0.815328 & 53480 & 34976 & 1,182 & 11057 \\
& DE & 1.354370 & 5.906805 & 0.814673 & 53752 & 35008 & 0,179 & 11095 \\
& ADE & 1.352783 & 5.913734 & 0.814175 & 53664 & 34992 & 0,190 & 11082 \\
& ADEPS & 1.359009 & 5.886643 & 0.814358 & 54096 & 34968 & 0,179 & 11133 \\
\hline
\end{tabular}

Table 13. Average BPP $P_{\text {code }}, C R_{\text {code }}, S S I M$ and \% difference against ADEPS of Polarimetric SAR image.

\begin{tabular}{lcccccc}
\hline & $\mathrm{BPP}_{\text {code }}$ & $\begin{array}{c}\text { \% difference } \\
\text { against ADEPS }\end{array}$ & $\mathrm{CR}_{\text {code }}$ & $\begin{array}{c}\text { \% difference } \\
\text { against ADEPS }\end{array}$ & SSIM & $\begin{array}{c}\text { \% difference } \\
\text { against ADEPS }\end{array}$ \\
\hline K-Means & 0.562570 & 1.17330 & 22.94952 & -4.09282 & 0.618264 & 1.060505 \\
DE & 0.567540 & 0.30022 & 22.46356 & -1.88863 & 0.621353 & 0.566179 \\
ADE & 0.569249 & 0 & 21.97433 & 0.330383 & 0.622288 & 0.416553 \\
ADEPS & 0.569249 & & 22.04717 & & 0.624891 & \\
\hline
\end{tabular}

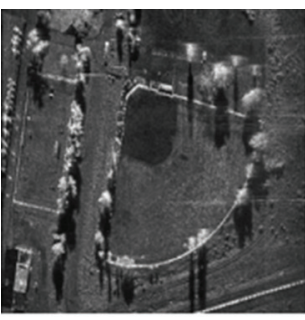

(a)

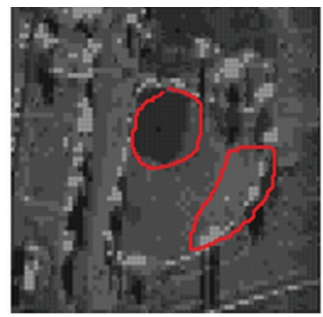

(b)

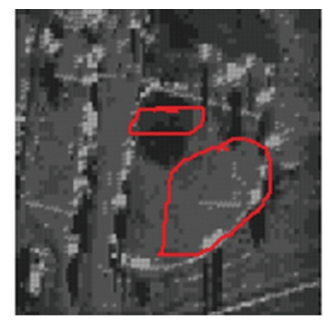

(c)

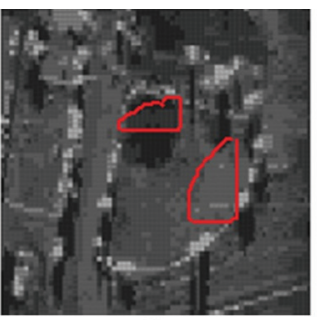

(d)

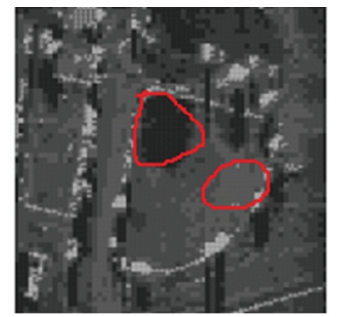

(e)

Fig. 17. Reconstructed Baseball image with a codebook size of 64 (a) Input Image (b) K-Means (c) DE (d) ADE (e) $h A D E-P S$. 


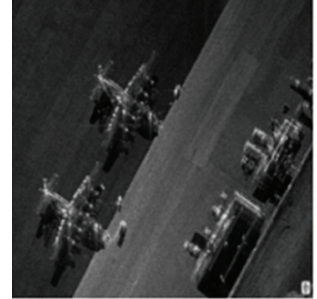

(a)

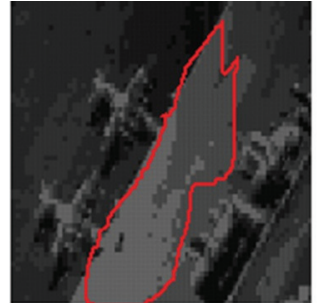

(b)

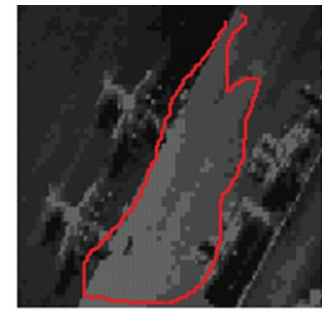

(c)

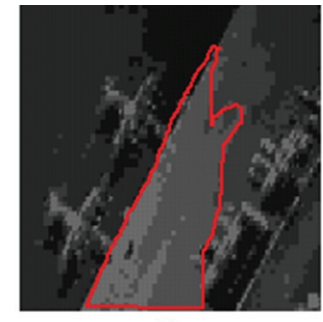

(d)

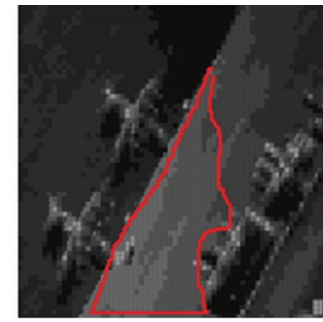

(e)

Fig. 18. Reconstructed Flight line SAR image with a codebook size of 16 (a) Input Image (b) K-Means (c) DE (d) $A D E$ (e) $h A D E-P S$.

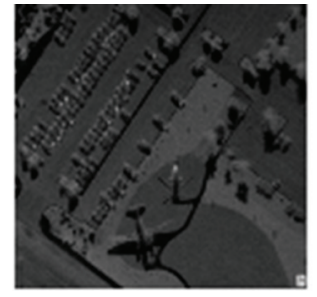

(a)

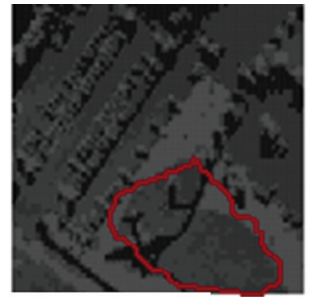

(b)

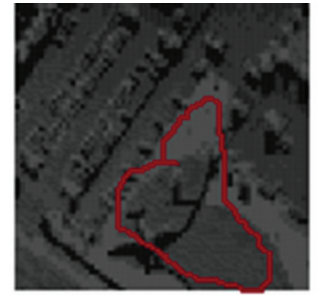

(c)

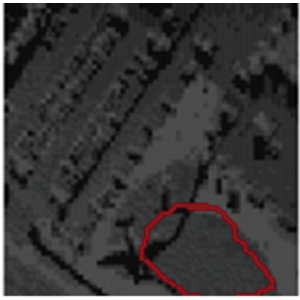

(d)

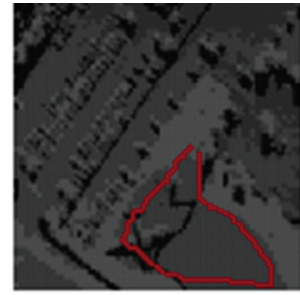

(e)

Fig. 19. Reconstructed Helicopter SAR image with a codebook size of 8 (a) Input Image (b) K-Means (c) DE (d) $A D E$ (e) hADE-PS.

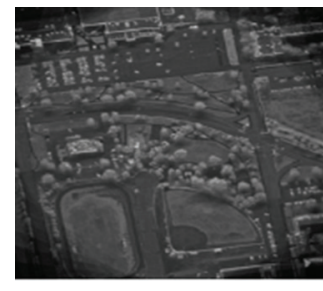

(a)

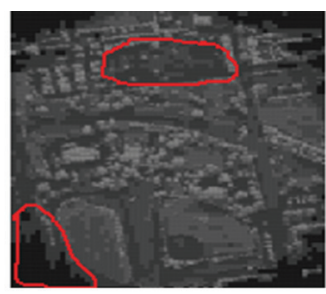

(b)

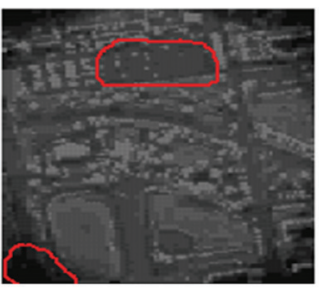

(c)

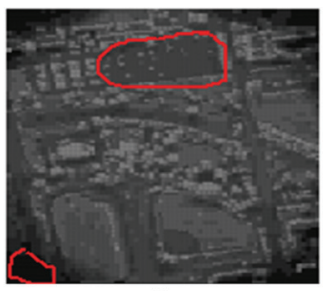

(d)

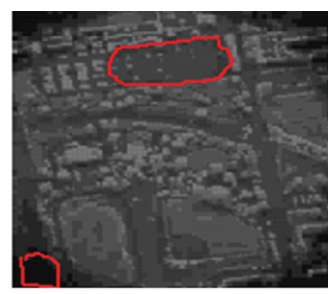

(e)

Fig. 20. Reconstructed Polarimetric SAR image with a codebook size of 8 (a) Input Image (b) K-Means (c) DE (d) $A D E$ (e) $h A D E-P S$.

\section{CONCLUSIONS}

In this paper, the performance analysis of different optimization techniques is compared which are used for optimizing the LBG vector quantization for efficient codebook design, that results in high image compression and good reconstructed image quality. Experimental results shows that hADE-PS has better performance in compression ratio and reconstructed image quality than DE, ADE and K-Means. Adaptive Differential Evolution algorithm gives true global minimum regardless of the initial parameter values with fast convergence, and with few control parameters.

\section{REFERENCES}

Abouali AH (2015). Object-based VQ for image compression. Ain Shams Engineering Journal, 6:211-16.

Chen Q, Yang JG, Gou J (2005). Image Compression Method by using Improved PSO Vector Quantization. Advances in Natural Computation, intr conf on neural computation (ICNC 2005), 490-95.

Chen Q (2012). Vector quantization method for image compression based on GA and LBG clustering algorithm. Computer Science 18:213-21.
Chiranjeevi K, Umaranjan J (2015). Fast vector quantization using a Bat algorithm for image compression. Engg Science and Tech, an Inter J 39:769-81.

Daubechies I (1988). Orthonormal basis of compactly supported wavelets. Comm. Pure Appl. Math., 12:909-96.

George ET, Dimitrios MT (2012). Fuzzy Clustering-Based Vector Quantization for Image Compression. Comp Intel in Image Proc, August, 93-105.

Hooke R, Jeeves TA. Direct search solution of numerical and statistical problems. J Assoc for Compu Machinery $8: 212-29$.

Horng MH, Jiang TW (2011). Image Vector Quantization Algorithm via Honey Bee Mating Optimization. Expert Sys with App, 38:1382-92.

Horng MH (2012). Vector Quantization using the firefly algorithm for Image Compression. Expert Sys with App 39:1078-91.

Hu YC, Su. BH, Tsou CC (2008). Fast VQ Codebook Search for Gray Scale Image Coding. Image and Vision Comp 26:657-66.

Krishna K, Ramakrishnan, Thathachar M (1997). Vector quantization using genetic k-means algorithm for image compression. Intr Conf on Infor, Comm and Signal Pro 3:1585-87. 
Linde Y, Buzo A, Gray RM (1980). An algorithm for vector quantize design. IEEE Tran on Comm 28:84-95.

Liu L, Ling C (2015). Polar Lattices are Good for Lossy Compression. IEEE Info Theory Workshop 342-46.

Patane G, Russo M. (2002). The enhanced LBG algorithm. Neural Networks 14:1219-37.

Poggi G, Ragozini ARP (2001). Tree-structured productcodebook vector quantization. Signal Proc: Img Comm 16:421-30

Price K, Storn RM, Lampinen J (2005). Differential evolution: A practical approach to global optimization. Natural Comp Ser Berlin: Springer, 732-41.

Rajpoot A, Hussain A, Saleem K, Qureshi Q (2004). A Novel Image Coding Algorithm Using Ant Colony System Vector Quantization. Intr workshop on sys, sig and img proc, Poznan, Poland 2:812-23.

Sanyal N, Chatterjee A, Munshi (2013). Modified bacterial foraging optimization technique for vector quantization-based image compression. Comp Int in Img Proc, Springer, Berlin, Heidelberg, 2:131-52.

Stron R, Price K (1995). Differential evolution: a simple and efficient adaptive scheme for global optimization over continuous spaces. J of Global Optimize 11:341-59.
Tsai CW, Tseng SP, Yang CS, Chiang MC (2013). PREACO: A Fast Ant Colony Optimization for Code-book Generation. Applied Soft Computing 13:3008-20.

Wang Y, Feng XY, Huang YX, Pu DB, Zhou WG, Liang YC (2007). A Novel Quantum Swarm Evolutionary Algorithm and its Applications. Neurocomputing 70: 633-40.

Yao X, Liu Y, Lin G (1999). Evolutionary programming made faster. IEEE Tran on Evol Compu 3:82-102.

Zeng Z, Cumming IG (2001). SAR Image Data Compression Using a Tree-Structured Wavelet Transform. IEEE Tran on Geosci and Remote Sen 39:546-52.

Zhao M, Liu H (2013). Vector quantization codebook design and application based on the clonal selection algorithm. Sensors and Transducers 159:415-21.

Zheng X, Julstrom BA, Cheng W (1997). Design of Vector Quantization Codebooks Using a Genetic Algorithm. IEEE Intr Conf on Evolutionary Comp, 13-16 April, University Place Hotel Indianapolis, USA, 525-29. 